\title{
PROJECT STATUS REPORT
}

$$
\text { DOE/PC/79997- } \overline{/ 10}
$$

May 18, 1990

Period Covered $1 / 9 / 90-4 / 8 / 90$

CONTRACT TITLE: Establishment and Maintenance of a Coal Sample Bank and Data Base

CONTRACT NUMBER: DE-RP22-87PC79997

DOE/PC/79997--T10

CONTRACTOR NAME: The Pennsylvania State University

517 Deike Building

DE92 015520

University Park, PA 16802

CONTRACT PERIOD: Apri1 1988 to April 1991

1. Contract Objective: No change

2. Technical Approach Changes: None

3. Contract Tasks:

Task 1-B: Storage of Coal Samples

\section{Documentation of Sample Quality and Deterioration}

Final analytical results were received from $5-1 b$ splits of $-1 / 4$ inch coal retrieved from designated drums in 1989. These results are shown in Appendix A.

Task 1-C: Collection of Samples, Sample Handling and Processing

Samples DECS-1 and DECS-2 collected in December were crushed (half of each to $-1 / 4^{\prime \prime}$ and half to -20 mesh) and placed in foil laminate bags for storage. Final splitting of the -20 mesh material into 256 lots for each sample is the only step remaining.

Task 1-D: Characterization of Coal Samples

Analytical results from DECS-1 and DECS-2 for total moisture, equilibrium moisture, proximate, ultimate, sulfur forms, calorific value, chlorine, and ash fusion were received. Epoxy-binder crushed particle pellets were prepared for reflectance and maceral analyses. Free-swelling index tests were run on both samples and alkali extraction and Gieseler fluidity tests were performed on DECS-2, the bituminous sample.

Task 1-E: Distribution of Samples to Users

During the current reporting period a total of 51 samples (36 DOE Sample Bank samples and 15 other Penn State samples) of various sizes were distributed. 
Task 2: Operation of the DOE Coal Data Base

1) During the current reporting period a total of 251 data printouts were distributed. In addition, 17 special data requests were fulfilled by either search/sort and printout or creation of a data disk, resulting in distribution of limited information on 2,989 samples. Several preliminary requests for Sample Eank and Data Base information and price quotations have also been handled.

2) Agencies supplied with coal samples and/or data include:

Abilene Christian University

American Lignite Co. (2)

Brigham Young University

Brown University

Cal Tech

CANMET Energy Mines \& Resources (Canada)

Chem. Eng.

Chevon 0 il Field Research Co.

Clark Atlanta University, Chemistry Department (2)

Coal Technology Corp. (2)

CONOCO Research Center

Engineering Resources

Indianapolis Power \& Light Co. (2)

Instituto Nacional del Carbon (Spain)

JK Research

Kentucky Center for Applied Energy Research

NIOSH

Northeastern University

Ohio Coal Development Office

Ohio University, Chemical Engineering Dept.

Penn State University, Fuel Science Dept. (3)

Penn State University, Geosciences Dept. (2)

Penn State University, Mining Engineering Dept. (2)

Penn State University, Mineral Processing Dept.

Science Ventures

Southern Illinois University Mechanical Engineering Dept.

Texaco Research Center

University of Alaska Mineral Industries Research Lab

University of British Columbia Geological Sciences Dept. (Canada)

University of Chicago, Chemistry Dept.

UNOCAL Science and Technology Division (2)

U.S. Department of Energy

U.S. Department of Energy, METC

U.S. Department of Energy, PETC

The numbers in parentheses are multiple requests which were separately processed.

DISCLAIMER

This report was prepared as an account of work sponsored by an agency of the United States Government. Neither the United States Government nor any agency thereof, nor any of their employees, makes any warranty, express or implied, or assumes any legal liability or responsibility for the accuracy, completeness, or usefulness of any information, apparatus, product, or process disclosed, or represents that its use would not infringe privately owned rights. Refer. ence herein to any specific commercial product, process, or service by trade name, tradernark, manufacturer, or otherwise does not necessarily constitute or imply its endorsement, recom-

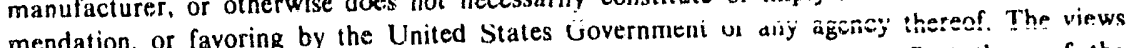
and opinions of authors expressed herein do not necessarily state or reflect those of the United States Government or any agency thereof. 
APPENDIX A

Reanalysis of DOE Sample Suite Coals

to Evaluate Sample Equivalence and Sample Integrity through April, 1990 


\begin{tabular}{|c|c|c|c|c|}
\hline $\begin{array}{ll}\text { PSOC }-1107 & K \\
\text { collection date: } & 0\end{array}$ & $\begin{array}{l}\text { KING CANNE! } \\
08 / 08 / 78\end{array}$ & \multicolumn{3}{|c|}{ subA, Ro max $=0.55$} \\
\hline $\begin{array}{r}\text { Split ID } \\
\text { size } \\
\% \text { oxygen } \\
\text { note } \\
\text { date sealed } \\
\text { date oxygen }\end{array}$ & $\begin{array}{l}\text { analysis } \\
\text { sample } 1\end{array}$ & $\begin{array}{l}\# 2 \text { can } \\
15 \text { of } 23 \\
-20 \text { mesh } \\
\text { a } \\
06 / 12 / 84\end{array}$ & \begin{tabular}{l}
30 gal. \\
1 of 1 \\
$-1 / 4^{11}$ \\
$-a$ \\
\hdashline$a$
\end{tabular} & $\begin{array}{l}30 \text { gal } \\
1 \text { of } 1 \\
-1 / 4^{\prime \prime} \\
18.6 \\
9 / 18 / 89\end{array}$ \\
\hline $\begin{array}{l}\text { PROXIMATE } \\
\% \text { moisture } \\
\% \text { ash (dry) } \\
\text { analysis date }\end{array}$ & $\begin{array}{c}7.7 \\
19.2 \\
11 / 04 / 83\end{array}$ & $\begin{array}{c}14.2 \\
19.4 \\
06 / 13 / 88\end{array}$ & $\begin{array}{c}11.2 \\
19.2 \\
3 / 07 / 89\end{array}$ & $\begin{array}{c}12.1 \\
19.2 \\
10 / 16 / 89\end{array}$ \\
\hline $\begin{array}{l}\text { SULFUR FORMS (dry \%) } \\
\text { total sulfur } \\
\text { sulfate } \\
\text { pyritic } \\
\text { organic } \\
\text { analysis date }\end{array}$ & $\begin{array}{c}2.16 \\
0.92 \\
0.43 \\
0.81 \\
11 / 04 / 83\end{array}$ & & $\begin{array}{c}1.71 \\
0.80 \\
0.45 \\
0.46 \\
3 / 07 / 89\end{array}$ & $\begin{array}{c}1.85 \\
0.84 \\
0.42 \\
0.59 \\
10 / 16 / 89\end{array}$ \\
\hline $\begin{array}{l}\text { CALORIFIC VALUE (dry) } \\
\text { BTU/lb } \\
\text { analysis date }\end{array}$ & $\begin{array}{c}10012 \\
11 / 04 / 83\end{array}$ & & $\begin{array}{c}9736 \\
3 / 07 / 89\end{array}$ & $\begin{array}{c}9565 \\
10 / 16 / 89\end{array}$ \\
\hline $\begin{array}{l}\text { ALKALI EXTRACTION } \\
\% \text { transmission } \\
\text { analysis date }\end{array}$ & & $\begin{array}{c}0.2 \\
06 / 13 / 88\end{array}$ & $\begin{array}{l}\text { discon- } \\
\text { tinued }\end{array}$ & \\
\hline
\end{tabular}

notes: a) Sample dry (dusty), visible yellow \& white sulfates iron stained, resealed in poly drum $11 / 16 / 88$. 


\begin{tabular}{|c|c|c|c|c|}
\hline $\begin{array}{l}\text { PSOC-1442 } \\
\text { collection date: } \\
\text { o }\end{array}$ & $\begin{array}{l}\text { DARCO } \\
03 / 28 / 85\end{array}$ & \multicolumn{3}{|c|}{ ligA, Ro $\max =0.4$} \\
\hline $\begin{array}{r}\text { Split ID } \\
\text { size } \\
\% \text { oxygen } \\
\text { note } \\
\text { date sealed } \\
\text { date oxygen } \\
\end{array}$ & $\begin{array}{l}\text { analysis } \\
\text { sample } 1 \\
-20 \text { mesh }\end{array}$ & $\begin{array}{c}30 \text { gal. } \\
3 \text { of } 3 \\
-1 / 4^{11} \\
3.16 \\
a \\
8 / 17 / 88\end{array}$ & $\begin{array}{l}30 \text { ga } 1 \\
3 \text { of } 3 \\
-1 / 4^{\prime \prime} \\
10.6 \\
9 / 18 / 89\end{array}$ & \\
\hline $\begin{array}{l}\text { PROXIMATE } \\
\% \text { moisture } \\
\% \text { ash (dry) } \\
\text { analysis date }\end{array}$ & \begin{tabular}{|c|}
32.6 \\
11.3 \\
$06 / 11 / 85$ \\
\end{tabular} & $\begin{array}{c}30.5 \\
9.4 \\
09 / 14 / 88\end{array}$ & $\begin{array}{c}34.1 \\
10.9 \\
10 / 16 / 89\end{array}$ & \\
\hline $\begin{array}{l}\text { SULFUR FORMS (dry \%) } \\
\text { total sulfur } \\
\text { sulfate } \\
\text { pyritic } \\
\text { organic } \\
\text { analysis date } \\
\end{array}$ & $\begin{array}{c}0.78 \\
0.02 \\
0.11 \\
0.65 \\
06 / 11 / 85 \\
\end{array}$ & $\begin{array}{c}1.05 \\
0.11 \\
0.10 \\
0.84 \\
09 / 22 / 88\end{array}$ & $\begin{array}{c}0.82 \\
0.08 \\
0.08 \\
0.66 \\
10 / 16 / 89\end{array}$ & \\
\hline $\begin{array}{l}\text { CALORIFIC VALUE (dry) } \\
\text { BTU/Ib } \\
\text { analysis date }\end{array}$ & $\begin{array}{c}11375 \\
06 / 11 / 85\end{array}$ & & $\begin{array}{c}11108 \\
10 / 16 / 89\end{array}$ & \\
\hline
\end{tabular}

notes: a) Sample moist, good condition, container resealed 08/17/88. PSU MCL. ash, moisture, sulfur forms. 


\begin{tabular}{|c|c|c|c|c|}
\hline \multicolumn{5}{|c|}{$\begin{array}{l}\text { PSOC- } 1443 \text { UNNAMED (Lower Wilcox) ligA, Ro } \max =0.44 \\
\text { collection date: } 03 / 29 / 85\end{array}$} \\
\hline $\begin{array}{r}\text { Split ID } \\
\text { size } \\
\% \text { head oxygen } \\
\text { note } \\
\text { date sealed } \\
\text { date oxygen }\end{array}$ & $\begin{array}{l}\text { analysis } \\
\text { sample } 1 \\
-20 \text { mesh }\end{array}$ & $\begin{array}{l}30 \text { gal. } \\
3 \text { of } 4 \\
-11 \\
3.84 \\
a \\
8 / 22 / 88\end{array}$ & $\begin{array}{l}30 \text { gal. } \\
3 \text { of } 4 \\
-1 / 4^{11} \\
6.8 \\
9 / 18 / 89\end{array}$ & \\
\hline $\begin{array}{l}\text { PROXIMATE } \\
\% \text { moisture } \\
\% \text { ash (dry) } \\
\text { analysis date }\end{array}$ & $\begin{array}{c}28.5 \\
21.4 \\
06 / 11 / 85\end{array}$ & $\begin{array}{c}29.2 \\
19.5 \\
09 / 14 / 88\end{array}$ & $\begin{array}{c}33.35 \\
20.97 \\
10 / 16 / 89\end{array}$ & \\
\hline $\begin{array}{l}\text { SULFUR FORMS (drJ \%) } \\
\text { total sulfur } \\
\text { sulfate } \\
\text { pyritic } \\
\text { organic } \\
\text { analysis date }\end{array}$ & $\begin{array}{c}0.75 \\
0.01 \\
0.09 \\
0.64 \\
06 / 11 / 85\end{array}$ & $\begin{array}{c}0.99 \\
0.03 \\
0.02 \\
0.94 \\
09 / 22 / 88\end{array}$ & $\begin{array}{c}0.83 \\
0.05 \\
0.02 \\
0.76 \\
10 / 16 / 89\end{array}$ & \\
\hline $\begin{array}{l}\text { CALORIFIC VALUE (dry) } \\
\text { BTU/1b } \\
\text { analysis date }\end{array}$ & $\begin{array}{c}9882 \\
06 / 11 / 85\end{array}$ & & $\begin{array}{c}9673 \\
10 / 16 / 89\end{array}$ & \\
\hline
\end{tabular}

notes: a) Good condition, moist, sample crushed to $-1 / 4^{\prime \prime}$ and resealed on $8 / 22 / 88$. PSU MCL ash, moisture, sulfur forms. 


\begin{tabular}{|c|c|c|c|c|}
\hline \multicolumn{5}{|c|}{$\begin{array}{l}\text { PSOC-1444 UNNAMED }(U . \text { Calvert B1.) ligA, Ro } \max =0.45 \\
\text { collection date: } 03 / 30 / 85\end{array}$} \\
\hline $\begin{array}{r}\text { Split ID } \\
\text { size } \\
\% \text { head oxygen } \\
\text { note } \\
\text { date sealed } \\
\text { date oxygen }\end{array}$ & $\begin{array}{l}\text { analysis } \\
\text { sample } 1 \\
-20 \text { mesh }\end{array}$ & $\begin{array}{l}\text { analysis } \\
\text { sample } 2\end{array}$ & $\begin{array}{c}30 \text { gal } \\
3 \text { of } 3 \\
-111 \\
1.35 \\
a \\
8 / 18 / 88\end{array}$ & $\begin{array}{l}30 \text { gal } \\
3 \text { of } 3 \\
-1 / 4^{\prime \prime} \\
9.8 \\
9 / 19 / 89\end{array}$ \\
\hline $\begin{array}{l}\text { PROXIMATE } \\
\% \text { motsture } \\
\% \text { ash (dry) } \\
\text { analysis date }\end{array}$ & $\begin{array}{c}31.2 \\
16.5 \\
06 / 11 / 85\end{array}$ & $\begin{array}{c}31.9 \\
16.8 \\
03 / 27 / 86\end{array}$ & $\begin{array}{c}28.2 \\
16.1 \\
09 / 14 / 88\end{array}$ & $\begin{array}{l}32.6 \\
16.9 \\
10 / 16 / 89\end{array}$ \\
\hline $\begin{array}{l}\text { SULFUR FORMS (dry \%) } \\
\text { total sulfur } \\
\text { sulfate } \\
\text { pyritic } \\
\text { organic } \\
\text { analysis date }\end{array}$ & $\begin{array}{c}0.96 \\
0.01 \\
0.05 \\
0.90 \\
06 / 11 / 85\end{array}$ & $03 / 27 / 86$ & $\begin{array}{c}1.19 \\
0.07 \\
0.19 \\
0.93 \\
09 / 22 / 88\end{array}$ & $\begin{array}{c}0.95 \\
0.13 \\
0.12 \\
0.70 \\
10 / 16 / 89\end{array}$ \\
\hline $\begin{array}{l}\text { CALORIFIC VALUE (dry) } \\
\text { BTU/lb } \\
\text { analysis date }\end{array}$ & $\begin{array}{c}10694 \\
06 / 11 / 85\end{array}$ & $\begin{array}{c}10817 \\
03 / 27 / 86\end{array}$ & & $\begin{array}{c}10463 \\
10 / 16 / 89\end{array}$ \\
\hline
\end{tabular}

notes: a) Trace of visible sulfates, moist, sample was crushed to $-1 / 4^{\prime \prime}$ and resealed on $8 / 18 / 88$. PSU MCL ash, moisture, sulfur forms. 


\begin{tabular}{|c|c|c|c|c|}
\hline $\begin{array}{l}\text { PSOC }-1445 \\
\text { collection date: }\end{array}$ & $\begin{array}{l}\text { BLUE \# } 1 \\
04 / 02 / 85\end{array}$ & \multicolumn{3}{|c|}{ huCb, Ro $\max =0.57$} \\
\hline $\begin{array}{r}\text { Split ID } \\
\text { size } \\
\text { \% head oxygen } \\
\text { note } \\
\text { date sealed } \\
\text { date oxygen }\end{array}$ & $\begin{array}{l}\text { analysis } \\
\text { sample } 1 \\
-20 \text { mesh }\end{array}$ & $\begin{array}{l}\# 2 \text { can } \\
15 \text { of } 19 \\
-20 \text { mesh } \\
05 / 02 / 85\end{array}$ & $\begin{array}{l}30 \text { gal } \\
2 \text { of } 3 \\
-111 \\
5.72 \\
\text { a } \\
8 / 22 / 88\end{array}$ & $\begin{array}{l}30 \text { gal } \\
2 \text { of } 3 \\
-1 / 4^{\prime \prime} \\
11.1 \\
9 / 19 / 89\end{array}$ \\
\hline $\begin{array}{l}\text { PROXIMATE } \\
\% \text { moisture } \\
\% \text { ash (dry) } \\
\text { analysis date }\end{array}$ & $\begin{array}{c}12.2 \\
4.6 \\
06 / 11 / 85\end{array}$ & $\begin{array}{c}13.8 \\
4.2 \\
06 / 13 / 88\end{array}$ & $\begin{array}{c}10.6 \\
4.8 \\
09 / 14 / 88\end{array}$ & $\begin{array}{l}14.1 \\
5.1 \\
10 / 16 / 89\end{array}$ \\
\hline $\begin{array}{l}\text { SULFUR FORMS (dry \%) } \\
\text { total sulfur } \\
\text { sulfate } \\
\text { pyritic } \\
\text { organic } \\
\text { analysis date }\end{array}$ & $\begin{array}{c}0.73 \\
0.01 \\
0.16 \\
0.55 \\
06 / 11 / 85\end{array}$ & & $\begin{array}{c}0.88 \\
0.17 \\
0.12 \\
0.49 \\
09 / 22 / 88\end{array}$ & $\begin{array}{c}0.85 \\
0.20 \\
0.12 \\
0.53 \\
10 / 16 / 89\end{array}$ \\
\hline $\begin{array}{l}\text { CALORIFIC VALUE (dry) } \\
\text { BTU/Ib } \\
\text { analysis date }\end{array}$ & $\begin{array}{c}13179 \\
06 / 11 / 85\end{array}$ & & & $\begin{array}{c}12837 \\
10 / 16 / 89\end{array}$ \\
\hline $\begin{array}{l}\text { ALKALI EXTRACTION } \\
\% \text { transmission } \\
\text { analysis date }\end{array}$ & & $\begin{array}{c}15.7 \\
06 / 13 / 88\end{array}$ & $\begin{array}{c}16.1 \\
09 / 22 / 88\end{array}$ & $\begin{array}{l}\text { discon- } \\
\text { tinued }\end{array}$ \\
\hline
\end{tabular}

notes: a) Good condition, moist, sample crushed to $-1 / 4^{\prime \prime}$ and resealed on $08 / 22 / 88$. PSU MCL ash, moisture, sulfur forms. 


\begin{tabular}{|c|c|c|c|c|c|}
\hline \multicolumn{6}{|c|}{$\begin{array}{cl}\text { PSOC-1446 } & \text { NEW MEXICO \#8 hvCb, Ro max }=0.59 \\
\text { collection date: } & 04 / 03 / 85\end{array}$} \\
\hline $\begin{array}{r}\text { Split ID } \\
\text { size } \\
\% \text { head oxygen } \\
\text { note } \\
\text { date sealed } \\
\text { date oxygen }\end{array}$ & $\begin{array}{l}\text { analysis } \\
\text { sample } 1 \\
-20 \text { mesh }\end{array}$ & $\begin{array}{l}\# 2 \text { can } \\
15 \text { of } 22 \\
-20 \text { mesh } \\
05 / 03 / 85\end{array}$ & $\begin{array}{c}30 \text { gal. } \\
3 \text { of } 3 \\
-1 / 4^{\prime \prime} \\
12.3 \\
\text { a }\end{array}$ & $\begin{array}{l}5 \mathrm{gal} \\
3 \text { of } 3 \\
-1 / 4^{\prime \prime} \\
20.6 \\
b \\
8 / 23 / 88 \\
\end{array}$ & $\begin{array}{l}30 \text { gal. } \\
3 \text { of } 3 \\
-1 / 4^{\prime \prime} \\
16.5 \\
9 / 19 / 89\end{array}$ \\
\hline $\begin{array}{l}\text { PROXIMATE } \\
\% \text { moisture } \\
\% \text { ash (dry) } \\
\text { analysis date } \\
\end{array}$ & $\begin{array}{c}11.2 \\
20.8 \\
06 / 10 / 85 \\
\end{array}$ & $\begin{array}{c}12.9 \\
19.9 \\
06 / 13 / 88 \\
\end{array}$ & $\begin{array}{r}9.7 \\
20.9 \\
09 / 14 / 88 \\
\end{array}$ & $\begin{array}{r}9.2 \\
21.2 \\
09 / 14 / 88 \\
\end{array}$ & $\begin{array}{c}12.2 \\
20.5 \\
10 / 16 / 89 \\
\end{array}$ \\
\hline $\begin{array}{l}\text { SULFUR FORMS (dry } \% \text { ) } \\
\text { total sulfur } \\
\text { sulfate } \\
\text { pyritic } \\
\text { organic } \\
\text { analysis date } \\
\end{array}$ & $\begin{array}{c}0.85 \\
0.01 \\
0.45 \\
0.39 \\
06 / 10 / 85 \\
\end{array}$ & & $\begin{array}{c}1.03 \\
0.18 \\
0.16 \\
0.69 \\
09 / 22 / 88 \\
\end{array}$ & $\begin{array}{c}1.00 \\
0.17 \\
0.17 \\
0.66 \\
09 / 22 / 88 \\
\end{array}$ & $\begin{array}{c}0.93 \\
0.21 \\
0.13 \\
0.60 \\
10 / 16 / 89 \\
\end{array}$ \\
\hline $\begin{array}{l}\text { CALORIFIC VALUE (dry) } \\
\text { BTU/lb } \\
\text { analysis date }\end{array}$ & $\begin{array}{c}10940 \\
06 / 10 / 85 \\
\end{array}$ & & & & $\begin{array}{c}10696 \\
10 / 16 / 89\end{array}$ \\
\hline $\begin{array}{l}\text { ALKALI EXTRACTION } \\
\% \text { transmission } \\
\text { analysis date }\end{array}$ & & $\begin{array}{c}38.3 \\
06 / 14 / 88 \\
\end{array}$ & $\begin{array}{c}37.1 \\
09 / 22 / 88\end{array}$ & & $\begin{array}{l}\text { discon- } \\
\text { tinued }\end{array}$ \\
\hline
\end{tabular}

notes: a) Good condition, resealed 08/23/88 (split 1). PSU MCL ash, moisture, sulfur forms.

b) white sulfate crystals visible on top surface. resealed on $08 / 23 / 88$ (split 2). PSU MCL ash, moisture, sulfur forms. 


\begin{tabular}{|c|c|c|c|c|}
\hline \multirow{2}{*}{$\begin{array}{l}\text { PSOC- } 1447 \\
\text { collection date: } \\
\text { Split ID } \\
\text { size } \\
\% \text { head oxygen } \\
\text { note } \\
\text { date sealed } \\
\text { date oxygen }\end{array}$} & \multicolumn{4}{|c|}{$\begin{array}{l}\text { RED } \\
04 / 04 / 85\end{array}$} \\
\hline & $\begin{array}{l}\text { analysis } \\
\text { sample } 1 \\
-60 \text { mesh }\end{array}$ & $\begin{array}{l}\# 2 \text { can } \\
15 \text { ? } 19 \\
-20 \text { mesh } \\
05 / 03 / 85\end{array}$ & $\begin{array}{c}30 \text { gal } \\
3 \text { of } 3 \\
-1 / 4^{\prime \prime} \\
13.0 \\
a \\
9 / 01 / 88\end{array}$ & $\begin{array}{l}30 \text { gal } \\
3 \text { of } 3 \\
-1 / 4^{11} \\
9 / 19 / 89\end{array}$ \\
\hline $\begin{array}{l}\text { PROXIMATE } \\
\% \text { moisture } \\
\% \text { ash (dry) } \\
\text { analysis date }\end{array}$ & $\begin{array}{c}10.1 \\
5.7 \\
06 / 10 / 85 \\
\end{array}$ & $\begin{array}{c}12.3 \\
5.1 \\
06 / 13 / 88 \\
\end{array}$ & $\begin{array}{c}10.1 \\
5.8 \\
10 / 12 / 88 \\
\end{array}$ & $\begin{array}{r}12.2 \\
6.1 \\
10 / 16 / 89 \\
\end{array}$ \\
\hline $\begin{array}{l}\text { SULFUR FORMS (dry \%) } \\
\text { total sulfur } \\
\text { sulfate } \\
\text { pyritic } \\
\text { organic } \\
\text { analysis date }\end{array}$ & $\begin{array}{c}0.41 \\
0.01 \\
0.23 \\
0.18 \\
06 / 10 / 85\end{array}$ & & $\begin{array}{c}0.39 \\
0.01 \\
0.04 \\
0.34 \\
01 / 17 / 89\end{array}$ & $\begin{array}{c}0.42 \\
0.02 \\
0.02 \\
0.38 \\
10 / 16 / 89\end{array}$ \\
\hline $\begin{array}{l}\text { CALORIFIC VALUE (dry) } \\
\text { BTU/Ib } \\
\text { analysis date }\end{array}$ & $\begin{array}{c}12983 \\
06 / 10 / 85\end{array}$ & & & $\begin{array}{c}12557 \\
10 / 16 / 89\end{array}$ \\
\hline $\begin{array}{l}\text { ALKALI EXTRACTION } \\
\text { \% transmission } \\
\text { analysis date }\end{array}$ & & $\begin{array}{c}5.6 \\
06 / 14 / 88\end{array}$ & $\begin{array}{c}4.5 \\
10 / 05 / 88\end{array}$ & $\begin{array}{l}\text { discon- } \\
\text { tinued }\end{array}$ \\
\hline
\end{tabular}

notes: Good condition, sample dry and dusty, lids loose on secondary buckets, sample resealed on 09/01/88. PSU MCL ash, moisture. PSU MCL sulfur forms (revised analys is report). 


\begin{tabular}{|c|c|c|c|c|}
\hline $\begin{array}{l}\text { PSOC-1448 } \\
\text { collection date: }\end{array}$ & \multicolumn{4}{|c|}{$\begin{array}{l}\text { YORK CANYON } \\
04 / 08 / 85\end{array}$} \\
\hline $\begin{array}{r}\text { Split ID } \\
\text { size } \\
\% \text { head oxygen } \\
\text { note } \\
\text { date sealed } \\
\text { date oxygen }\end{array}$ & $\begin{array}{l}\text { anaiysis } \\
\text { sample } 1 \\
-20 \text { mesh }\end{array}$ & $\begin{array}{l}\# 2 \text { can } \\
8 \text { of } 29 \\
-20 \text { mesh } \\
\\
05 / 07 / 85\end{array}$ & $\begin{array}{l}30 \text { gal. } \\
3 \text { of } 3 \\
-1 / 4^{\prime \prime} \\
18.7 \\
\text { a } \\
8 / 29 / 88\end{array}$ & $\begin{array}{l}30 \mathrm{gal} \\
3 \text { of } 3 \\
-1 / 4^{11} \\
19.5 \\
9 / 19 / 89\end{array}$ \\
\hline $\begin{array}{l}\text { PROXIMATE } \\
\% \text { moisture } \\
\% \text { ash (dry) } \\
\text { analysis date } \\
\end{array}$ & $\begin{array}{c}1.6 \\
11.4 \\
06 / 10 / 85 \\
\end{array}$ & $\begin{array}{c}2.2 \\
11.1 \\
04 / 04 / 88\end{array}$ & $\begin{array}{c}1.1 \\
11.3 \\
09 / 19 / 88\end{array}$ & $\begin{array}{c}1.9 \\
11.1 \\
10 / 16 / 89\end{array}$ \\
\hline $\begin{array}{l}\text { GIESELER FLUIDITY } \\
\text { max. ddpm } \\
\text { fluid range } \\
\text { analysis date }\end{array}$ & $\begin{array}{r}5632 \\
72 \\
06 / 03 / 85 \\
\end{array}$ & $\begin{array}{r}2661 \\
89 \\
04 / 04 / 88 \\
\end{array}$ & $\begin{array}{c}659 \\
74 \\
09 / 08 / 88 \\
\end{array}$ & $\begin{array}{c}230 \\
74 \\
02 / 21 / 90\end{array}$ \\
\hline $\begin{array}{l}\text { SULFUR FORMS (dry \%) } \\
\text { total sulfur } \\
\text { sulfate } \\
\text { pyritic } \\
\text { organic } \\
\text { analysis date }\end{array}$ & $\begin{array}{c}0.46 \\
0.01 \\
0.04 \\
0.41 \\
06 / 10 / 85\end{array}$ & & $\begin{array}{c}0.49 \\
0.02 \\
0.05 \\
0.42 \\
01 / 17 / 89\end{array}$ & $\begin{array}{c}0.46 \\
0.03 \\
0.03 \\
0.40 \\
10 / 16 / 89\end{array}$ \\
\hline $\begin{array}{l}\text { CALORIFIC VALUE (dry) } \\
\text { BTU/lb } \\
\text { analysis date }\end{array}$ & $\begin{array}{c}13467 \\
06 / 10 / 85\end{array}$ & & & $\begin{array}{c}13198 \\
10 / 16 / 89\end{array}$ \\
\hline $\begin{array}{c}\text { ALKALI EXTRACTION } \\
\% \text { transmission } \\
\text { analysis date }\end{array}$ & & $\begin{array}{c}100.1 \\
06 / 01 / 88\end{array}$ & $\begin{array}{c}97.7 \\
09 / 23 / 88\end{array}$ & \\
\hline
\end{tabular}

notes: a) PSU MCL ash, moisture. PSU MCL sulfur forms (revised analysis report). 


\begin{tabular}{|c|c|c|c|c|c|c|}
\hline $\begin{array}{l}\text { PSOC-1451 } \\
\text { collection date: }\end{array}$ & $\begin{array}{l}\text { ITTSBURGH } \\
5 / 01 / 85\end{array}$ & hYAb, & Ro $\max =0$ & .77 & & \\
\hline $\begin{array}{r}\text { Split ID } \\
\text { size } \\
\% \text { head oxygen } \\
\text { note } \\
\text { date sealed } \\
\text { date oxygen }\end{array}$ & $\begin{array}{l}\text { analysis } \\
\text { sample } 1 \\
-20 \text { mesh }\end{array}$ & $\begin{array}{l}\text { analysis } \\
\text { sample } 2 \\
-20 \text { mesh }\end{array}$ & $\begin{array}{l}\text { analysis } \\
\text { sample } 3 \\
-20 \text { mesh }\end{array}$ & $\mid \begin{array}{c}\# 2 \text { can } \\
1 \text { of } ? \\
-20 \text { mesh } \\
11 / 03 / 87\end{array}$ & $\begin{array}{l}5 \mathrm{gal} \\
-1 / 4^{\prime \prime} \\
\text { a }\end{array}$ & $\begin{array}{l}30 \text { gal. } \\
1 \text { of } 3 \\
-1 / 4^{\prime \prime} \\
14.6 \\
9 / 20 / 89\end{array}$ \\
\hline $\begin{array}{l}\text { PROXIMATE } \\
\% \text { moisture } \\
\% \text { ash (dry) } \\
\text { analysis date }\end{array}$ & $\begin{array}{c}2.6 \\
14.2 \\
06 / 11 / 85\end{array}$ & $\begin{array}{c}2.5 \\
13.7 \\
02 / 14 / 86\end{array}$ & $\begin{array}{c}2.5 \\
13.9 \\
02 / 18 / 86\end{array}$ & $\begin{array}{c}3.6 \\
13.9 \\
04 / 06 / 88\end{array}$ & $\begin{array}{c}1.8 \\
13.4 \\
09 / 14 / 88\end{array}$ & $\begin{array}{l}\text { discon- } \\
\text { tinued }\end{array}$ \\
\hline $\begin{array}{l}\text { GIESELER FLUIDITY } \\
\text { max. ddpm } \\
\text { fluid range } \\
\text { analysis date }\end{array}$ & $\begin{array}{r}8525 \\
80 \\
05 / 29 / 85 \\
\end{array}$ & & & $\begin{array}{r}59 \\
62 \\
04 / 06 / 88 \\
\end{array}$ & $\begin{array}{c}225 \\
63 \\
08 / 17 / 88 \\
\end{array}$ & $\begin{array}{l}\text { to be } \\
\text { resamp- } \\
\text { led }\end{array}$ \\
\hline $\begin{array}{l}\text { SULFUR FORMS (dry \%) } \\
\text { total sulfur } \\
\text { sulfate } \\
\text { pyritic } \\
\text { organic } \\
\text { analysis date } \\
\end{array}$ & $\begin{array}{c}1.36 \\
0.01 \\
0.82 \\
0.53 \\
06 / 10 / 85 \\
\end{array}$ & $\begin{array}{r}1.37 \\
02 / 12 / 86 \\
\end{array}$ & $\begin{array}{r}1.38 \\
02 / 18 / 86 \\
\end{array}$ & & $\begin{array}{l}1.39 \\
0.15 \\
0.40 \\
0.84 \\
09 / 22 / 88 \\
\end{array}$ & \\
\hline $\begin{array}{l}\text { CALORIFIC VALUE (dry) } \\
\text { BTU/Ib } \\
\text { analysis date }\end{array}$ & $\begin{array}{c}12863 \\
06 / 10 / 85\end{array}$ & $\begin{array}{c}12855 \\
02 / 12 / 86\end{array}$ & $\begin{array}{c}12893 \\
02 / 18 / 86\end{array}$ & & & \\
\hline $\begin{array}{c}\text { ALKALI EXTRACTION } \\
\% \text { transmission } \\
\text { analysis date }\end{array}$ & & & & $\begin{array}{c}99.5 \\
06 / 02 / 88\end{array}$ & & \\
\hline
\end{tabular}

notes: a) Sample used for restocking, entire sample ground to -20 mesh. PSU MCL ash, moisture, sulfur forms. 


\begin{tabular}{|l|c|c|c|c|}
\hline $\begin{array}{c}\text { PSOC-1461 } \\
\text { collection date: }\end{array}$ MAMMOTH \\
$05 / 24 / 85$
\end{tabular}

notes: a) Good condition, sample dry. PSU MCL ash, moisture. PSU MCL sulfur forms (revised analysis report). 


\begin{tabular}{|c|c|c|c|c|}
\hline $\begin{array}{l}\text { PSOC }-1468 \\
\text { collection date: }\end{array}$ & $\begin{array}{l}\text { UCK MOUNTA } \\
6 / 03 / 85\end{array}$ & IN an, & $\max =5$ & \\
\hline $\begin{array}{r}\text { Split ID } \\
\text { size } \\
\% \text { head oxygen } \\
\text { note } \\
\text { date sealed } \\
\text { date oxygen }\end{array}$ & $\begin{array}{l}\text { analysis } \\
\text { sample } 1 \\
-60 \text { mesh }\end{array}$ & $\begin{array}{l}30 \text { gal } \\
1 \text { of } 3 \\
-1 / 4^{11} \\
17.8 \\
a \\
8 / 07 / 85 \\
9 / 25 / 88\end{array}$ & $\begin{array}{l}30 \text { gal } \\
1 \text { of } 3 \\
-1 / 4^{11} \\
20.3 \\
9 / 28 / 89\end{array}$ & \\
\hline $\begin{array}{l}\text { PROXIMATE } \\
\% \text { moistire } \\
\% \text { ash (dry) } \\
\text { analysis date }\end{array}$ & $\begin{array}{c}4.5 \\
6.8 \\
10 / 08 / 85 \\
\end{array}$ & $\begin{array}{c}3.5 \\
6.6 \\
10 / 12 / 88\end{array}$ & $\begin{array}{c}4.6 \\
6.6 \\
12 / 14 / 89\end{array}$ & \\
\hline $\begin{array}{l}\text { SULFUR FORMS (dry \%) } \\
\text { total sulfur } \\
\text { sulfate } \\
\text { pyritic } \\
\text { organic } \\
\text { analysis date }\end{array}$ & $\begin{array}{c}0.49 \\
0.01 \\
0.11 \\
0.37 \\
10 / 08 / 85\end{array}$ & $\begin{array}{c}0.43 \\
0.01 \\
0.07 \\
0.35 \\
01 / 17 / 89\end{array}$ & $\begin{aligned} 0.39 \\
0.03 \\
0.06 \\
0.29 \\
12 / 14 / 89\end{aligned}$ & \\
\hline $\begin{array}{l}\text { CALORIFIC VALUE (dry) } \\
\text { BTU/Ib } \\
\text { analysis date }\end{array}$ & $\begin{array}{c}13748 \\
10 / 08 / 85\end{array}$ & & $\begin{array}{c}13671 \\
12 / 14 / 89\end{array}$ & \\
\hline
\end{tabular}

notes: a) Sample dry, good condition. PSU MCL ash, moisture. PSU MCL sulfur forms (revised analysis report). 


\begin{tabular}{|c|c|c|c|c|}
\hline $\begin{array}{l}\text { PSOC }-1469 \\
\text { collection date: }\end{array}$ & $\begin{array}{l}\text { MARY LEE } \\
06 / 10 / 85\end{array}$ & \multicolumn{3}{|c|}{$h \vee B b$, Ro $\max =1.12$} \\
\hline $\begin{array}{r}\text { Spitt ID } \\
\text { size } \\
\% \text { head oxygen } \\
\text { note } \\
\text { date sealed } \\
\text { date oxygen }\end{array}$ & $\begin{array}{l}\text { analysis } \\
\text { sample } 1 \\
-60 \text { mesh }\end{array}$ & $\begin{array}{l}\# 2 \text { can } \\
26 \text { of } 31 \\
-20 \text { mesh } \\
10 / 01 / 85\end{array}$ & $\begin{array}{l}30 \text { gal. } \\
3 \text { of } 3 \\
-1 / 4^{\prime \prime} \\
15.0 \\
\text { a } \\
8 / 29 / 88\end{array}$ & $\begin{array}{l}30 \text { gal. } \\
3 \text { of } 3 \\
-1 / 4^{\prime \prime} \\
18.1 \\
9 / 28 / 89\end{array}$ \\
\hline $\begin{array}{l}\text { PROXIMATE } \\
\% \text { molsture } \\
\% \text { ash (dry) } \\
\text { analysis date } \\
\end{array}$ & $\begin{array}{c}1.9 \\
21.0 \\
10 / 08 / 85 \\
\end{array}$ & $\begin{array}{r}1.9 \\
21.1 \\
04 / 08 / 88 \\
\end{array}$ & $\begin{array}{c}1.3 \\
20.7 \\
09 / 19 / 88 \\
\end{array}$ & $\begin{array}{c}2.3 \\
20.8 \\
02 / 06 / 90\end{array}$ \\
\hline $\begin{array}{l}\text { GIESELER FLUIDITY } \\
\text { max. ddpm } \\
\text { fluid range } \\
\text { analysis date }\end{array}$ & $\begin{array}{r}659 \\
73 \\
09 / 11 / 85\end{array}$ & $\begin{array}{c}189 \\
71 \\
04 / 07 / 88\end{array}$ & $\begin{array}{c}21 \\
64 \\
09 / 09 / 88\end{array}$ & $\begin{array}{c}8 \\
54 \\
01 / 03 / 90\end{array}$ \\
\hline $\begin{array}{l}\text { SULFUR FORMS (dry \%) } \\
\text { total sulfur } \\
\text { sulfate } \\
\text { pyritic } \\
\text { organic } \\
\text { analysis date }\end{array}$ & $\begin{array}{c}0.80 \\
0.02 \\
0.26 \\
0.52 \\
10 / 08 / 85\end{array}$ & & $\begin{array}{c}0.84 \\
0.06 \\
0.14 \\
0.64 \\
01 / 17 / 89\end{array}$ & $\begin{array}{c}0.78 \\
0.07 \\
0.13 \\
0.58 \\
02 / 06 / 90\end{array}$ \\
\hline $\begin{array}{l}\text { CALORIFIC VALUE (dry) } \\
\text { BTU/Ib } \\
\text { analysis date }\end{array}$ & $\begin{array}{c}11838 \\
10 / 08 / 85\end{array}$ & & & $\begin{array}{c}11742 \\
02 / 06 / 90 \\
\end{array}$ \\
\hline $\begin{array}{c}\text { ALKALI EXTRACTION } \\
\% \text { transmission } \\
\text { analysis date }\end{array}$ & & $\begin{array}{c}99.8 \\
06 / 02 / 88\end{array}$ & $\begin{array}{c}95.5 \\
09 / 23 / 88\end{array}$ & $\begin{array}{l}94.4 \\
01 / 03 / 90\end{array}$ \\
\hline
\end{tabular}

notes: a) Good condition, sample dry. PSU MCL ash, moisture. PSU MCL sulfur forms (revised analysis report). 


\begin{tabular}{|c|c|c|c|c|c|}
\hline $\begin{array}{l}\text { PSOC-1470 } \\
\text { collection date: }\end{array}$ & $\begin{array}{l}\text { RATT } \\
6 / 12 / 85\end{array}$ & $h v B b$, & Ro $\max =0$ & .89 & \\
\hline $\begin{array}{r}\text { Split ID } \\
\text { size } \\
\% \text { head oxygen } \\
\text { note } \\
\text { date sealed } \\
\text { date oxygen }\end{array}$ & $\begin{array}{l}\text { analysis } \\
\text { sample } 1 \\
-60 m e s h\end{array}$ & $\begin{array}{l}\# 2 \text { can } \\
19 \text { of } 33 \\
-20 \text { mesh } \\
10 / 29 / 85\end{array}$ & $\begin{array}{l}30 \text { gal } \\
2 \text { of } 2 \\
-111 \\
8.6 \\
a\end{array}$ & $\begin{array}{l}5 \text { gal } \\
2 \text { of } 4 \\
-1 / 4^{\prime \prime} \\
17.9 \\
b \\
8 / 29 / 88\end{array}$ & $\begin{array}{l}30 \text { gal } \\
2 \text { of } 2 \\
-1 / 4^{11} \\
9.3 \\
9 / 28 / 89\end{array}$ \\
\hline $\begin{array}{l}\text { PROXIMATE } \\
\% \text { moisture } \\
\% \text { ash (dry) } \\
\text { analysis date }\end{array}$ & $\begin{array}{c}2.9 \\
20.6 \\
10 / 08 / 85\end{array}$ & $\begin{array}{c}3.0 \\
21.0 \\
04 / 14 / 88\end{array}$ & $\begin{array}{c}2.5 \\
20.5 \\
09 / 19 / 88\end{array}$ & $\begin{array}{c}3.1 \\
20.3 \\
09 / 19 / 88\end{array}$ & $\begin{array}{c}3.6 \\
20.6 \\
2 / 28 / 90\end{array}$ \\
\hline $\begin{array}{l}\text { GIESELER FLUIDITY } \\
\text { max. ddpm } \\
\text { fluid range } \\
\text { analysis date }\end{array}$ & $\begin{array}{r}487 \\
66 \\
10 / 11 / 85\end{array}$ & $\begin{array}{c}3 \\
33 \\
04 / 14 / 88\end{array}$ & $\begin{array}{c}55 \\
56 \\
09 / 12 / 88\end{array}$ & & $\begin{array}{l}4 \\
38 \\
2 / 28 / 90\end{array}$ \\
\hline $\begin{array}{l}\text { SULFUR FORMS (dry \%) } \\
\text { total sulfur } \\
\text { sulfate } \\
\text { pyritic } \\
\text { organic } \\
\text { analysis date }\end{array}$ & $\begin{array}{c}2.50 \\
0.04 \\
1.88 \\
0.58 \\
10 / 08 / 85\end{array}$ & & $\begin{array}{c}2.56 \\
0.37 \\
1.16 \\
1.03 \\
01 / 17 / 89\end{array}$ & $\begin{array}{c}2.58 \\
0.62 \\
0.96 \\
1.00 \\
01 / 17 / 89\end{array}$ & $\begin{array}{c}2.48 \\
0.47 \\
1.11 \\
0.90 \\
2 / 28 / 90\end{array}$ \\
\hline $\begin{array}{l}\text { CALORIFIC VALUE (dry) } \\
\text { BTLIIb } \\
\text { analysis date }\end{array}$ & $\begin{array}{c}11599 \\
10 / 08 / 85\end{array}$ & & & & $\begin{array}{c}11458 \\
2 / 28 / 90\end{array}$ \\
\hline $\begin{array}{l}\text { ALKALI EXTRACTION } \\
\% \text { transmission } \\
\text { analysis date }\end{array}$ & & $\begin{array}{c}98.8 \\
06 / 03 / 88\end{array}$ & $\begin{array}{c}95.8 \\
09 / 32 / 88\end{array}$ & & $\begin{array}{c}92.9 \\
2 / 22 / 90\end{array}$ \\
\hline
\end{tabular}

notes: a) Sample molst, trace of yellow sulfate on top surface sample was crushed to -1" and then split, plastometer data fluid range estimated.

PSU MCL ash, moisture. PSU MCL sulfur forms (revised analysis report).

b) white fibrous sulfate visible on surface. PSU MCL ash, moisture. PSU MCL sulfur forms (revised analysis report). 


\begin{tabular}{|c|c|c|c|c|}
\hline \multirow{2}{*}{\begin{tabular}{|c} 
PSOC-1471 \\
collection date: \\
Split IO \\
size \\
$\%$ head oxygen \\
note \\
date sealed \\
date oxygen
\end{tabular}} & $\begin{array}{l}\text { PEE WEE } \\
06 / 13 / 85\end{array}$ & \multicolumn{3}{|c|}{ hvAb, Ro $\max =0.94$} \\
\hline & $\begin{array}{l}\text { analysis } \\
\text { sample } 1 \\
-60 \text { mesh }\end{array}$ & $\begin{array}{l}\# 2 \text { can } \\
20 \text { of } 37 \\
-20 \text { mesh } \\
10 / 29 / 85\end{array}$ & $\begin{array}{c}30 \text { gal. } \\
1 \text { of } 3 \\
-1 / 4^{11} \\
15.7 \\
a \\
08 / 21 / 85 \\
8 / 30 / 88\end{array}$ & $\begin{array}{l}30 \text { gal. } \\
1 \text { of } 3 \\
-1 / 4^{\prime \prime} \\
20.3 \\
9 / 28 / 89\end{array}$ \\
\hline $\begin{array}{l}\text { PROXIMATE } \\
\% \text { moisture } \\
\% \text { ash (dry) } \\
\text { analysis date }\end{array}$ & $\begin{array}{c}2.5 \\
8.0 \\
10 / 08 / 85\end{array}$ & $\begin{array}{c}2.7 \\
7.7 \\
04 / 15 / 88\end{array}$ & $\begin{array}{c}2.2 \\
7.9 \\
10 / 12 / 88\end{array}$ & $\begin{array}{r}3.0 \\
8.0 \\
4 / 17 / 90 \\
\end{array}$ \\
\hline $\begin{array}{l}\text { GIESELER FLUIDITY } \\
\text { max. ddpm } \\
\text { fluid range } \\
\text { analysis date }\end{array}$ & $\begin{array}{r}90 \\
60 \\
10 / 16 / 85\end{array}$ & $\begin{array}{r}116 \\
66 \\
04 / 15 / 88\end{array}$ & $\begin{array}{c}3 \\
39 \\
10 / 14 / 88\end{array}$ & $\begin{array}{c}2 \\
22 \\
2 / 23 / 90\end{array}$ \\
\hline $\begin{array}{l}\text { SULFUR FORMS (dry \%) } \\
\text { total sulfur } \\
\text { sulfate } \\
\text { pyritic } \\
\text { organic } \\
\text { analysis date }\end{array}$ & $\begin{array}{c}1.04 \\
0.03 \\
0.22 \\
0.79 \\
10 / 08 / 85\end{array}$ & & $\begin{array}{c}1.01 \\
0.10 \\
0.13 \\
0.78 \\
01 / 17 / 89\end{array}$ & $\begin{array}{c}1.06 \\
0.11 \\
0.11 \\
0.84 \\
4 / 17 / 90\end{array}$ \\
\hline $\begin{array}{l}\text { CALORIFIC VALUE (dry) } \\
\text { BTU/Ib } \\
\text { analysis date }\end{array}$ & $\begin{array}{c}13715 \\
10 / 08 / 85\end{array}$ & & & $\begin{array}{c}13539 \\
4 / 17 / 90\end{array}$ \\
\hline $\begin{array}{l}\text { ALKALI EXTRACTION } \\
\% \text { transmission } \\
\text { analysis date }\end{array}$ & & $\begin{array}{c}94.7 \\
06 / 03 / 88\end{array}$ & $\begin{array}{c}97 \cdot 2 \\
10 / 05 / 88\end{array}$ & $\begin{array}{c}87.1 \\
2 / 23 / 90\end{array}$ \\
\hline
\end{tabular}

notes: a) Dry, good condition. PSU MCL ash, moisture. PSU MCL sulfur forms (revised analysis report). 


\begin{tabular}{|c|c|c|c|c|}
\hline $\begin{array}{l}\text { PSOC- } 1474 \\
\text { collection date: }\end{array}$ & $\begin{array}{l}\text { PLASHDAM } \\
6 / 17 / 85\end{array}$ & mub, & Ro $\max =$ & \\
\hline $\begin{array}{r}\text { Split ID } \\
\text { size } \\
\% \text { head oxygen } \\
\text { note } \\
\text { date sealed } \\
\text { date oxygen }\end{array}$ & $\begin{array}{l}\text { analysis } \\
\text { sample } 1 \\
-60 \text { mesh }\end{array}$ & $\begin{array}{l}\# 2 \text { can } \\
26 \text { of } 33 \\
-20 \text { mesh } \\
10 / 29 / 85\end{array}$ & $\begin{array}{c}30 \text { gal } \\
3 \text { of } 3 \\
-1 / 411 \\
16.3 \\
a \\
08 / 27 / 85 \\
8 / 30 / 88\end{array}$ & $\begin{array}{l}30 \text { gal. } \\
3 \text { of } 3 \\
-1 / 4^{\prime \prime} \\
16.3 \\
9 / 28 / 89\end{array}$ \\
\hline $\begin{array}{l}\text { PROXIMATE } \\
\% \text { moisture } \\
\% \text { ash (dry) } \\
\text { analysis date }\end{array}$ & $\begin{array}{c}1.6 \\
12.4 \\
10 / 22 / 85\end{array}$ & $\begin{array}{c}1.1 \\
13.4 \\
04 / 18 / 88\end{array}$ & $\begin{array}{c}1.2 \\
12.3 \\
10 / 12 / 88\end{array}$ & $\begin{array}{c}1.9 \\
12.4 \\
2 / 28 / 90\end{array}$ \\
\hline $\begin{array}{l}\text { GIESELER FLUIDITY } \\
\text { max. ddpm } \\
\text { fluid range } \\
\text { analysis date }\end{array}$ & $\begin{array}{c}14490 \\
99 \\
09 / 17 / 85\end{array}$ & $\begin{array}{c}743 \\
83 \\
04 / 18 / 88\end{array}$ & $\begin{array}{c}556 \\
84 \\
10 / 18 / 88\end{array}$ & $\begin{array}{c}310 \\
78 \\
2 / 28 / 90\end{array}$ \\
\hline $\begin{array}{l}\text { SULFUR FORMS (dry \%) } \\
\text { tctal sulfur } \\
\text { sulfate } \\
\text { pyritic } \\
\text { organic } \\
\text { analysis date }\end{array}$ & $\begin{array}{c}0.73 \\
0.01 \\
0.16 \\
0.56 \\
10 / 22 / 85\end{array}$ & & $\begin{array}{c}0.70 \\
0.05 \\
0.11 \\
0.54 \\
01 / 17 / 89\end{array}$ & $\begin{array}{c}0.68 \\
0.07 \\
0.07 \\
0.54 \\
4 / 17 / 90\end{array}$ \\
\hline $\begin{array}{l}\text { CALORIFIC VALUE (dry) } \\
\text { BTU/lb } \\
\text { analysis date }\end{array}$ & $\begin{array}{c}13588 \\
10 / 22 / 85\end{array}$ & & & $\begin{array}{c}13428 \\
4 / 17 / 90\end{array}$ \\
\hline $\begin{array}{l}\text { ALKALI EXTRACTION } \\
\% \text { transmission } \\
\text { analysis date }\end{array}$ & & $\begin{array}{c}98.5 \\
06 / 03 / 88\end{array}$ & $\begin{array}{c}98.9 \\
10 / 05 / 88\end{array}$ & $\begin{array}{c}92.6 \\
2 / 28 / 90\end{array}$ \\
\hline
\end{tabular}

notes: a) Dry, good condition. PSU MCL ash, moisture. PSU MCL sulfur forms (revised analysis report). 


\begin{tabular}{|c|c|c|c|c|}
\hline $\begin{array}{l}\text { PSOC- } 1475 \\
\text { collection date: }\end{array}$ & $\begin{array}{l}\text { Tkhorn \#3 } \\
6 / 18 / 85\end{array}$ & hvAb, & Ro $\max =0$ & \\
\hline $\begin{array}{r}\text { Split ID } \\
\text { size } \\
\% \text { head oxygen } \\
\text { note } \\
\text { date sealed } \\
\text { date oxygen }\end{array}$ & $\begin{array}{l}\text { analysis } \\
\text { sample } 1 \\
-60 \text { mesh }\end{array}$ & $\begin{array}{l}\# 2 \text { can } \\
27 \text { of } 33 \\
-20 \text { mesh } \\
10 / 29 / 85\end{array}$ & $\begin{array}{c}30 \text { gal. } \\
4 \text { of } 4 \\
-1 / 4^{\prime \prime} \\
9.5 \\
a \\
08 / 28 / 88 \\
9 / 01 / 88\end{array}$ & $\begin{array}{l}30 \text { gal. } \\
4 \text { of } 4 \\
-1 / 4^{\prime \prime} \\
13.5 \\
9 / 28 / 89\end{array}$ \\
\hline $\begin{array}{l}\text { PROXIMATE } \\
\% \text { moisture } \\
\% \text { ash (dry) } \\
\text { analysis date }\end{array}$ & $\begin{array}{c}3.4 \\
3.3 \\
10 / 23 / 85\end{array}$ & $\begin{array}{c}4.3 \\
1.5 \\
04 / 19 / 88\end{array}$ & $\begin{array}{c}2.9 \\
2.9 \\
10 / 12 / 88\end{array}$ & $\begin{array}{c}3.5 \\
3.2 \\
4 / 17 / 90\end{array}$ \\
\hline $\begin{array}{l}\text { GIESELER FLUIDITY } \\
\text { max. ddpm } \\
\text { fluid range } \\
\text { analysis date }\end{array}$ & $\begin{array}{r}177 \\
59 \\
09 / 18 / 85\end{array}$ & $\begin{array}{c}15 \\
59 \\
04 / 19 / 88\end{array}$ & $\begin{array}{c}3 \\
33 \\
10 / 20 / 88\end{array}$ & $\begin{array}{c}2 \\
30 \\
3 / 02 / 90\end{array}$ \\
\hline $\begin{array}{l}\text { SULFUR FORMS (dry \%) } \\
\text { total sulfur } \\
\text { sulfate } \\
\text { pyritic } \\
\text { organic } \\
\text { analysis date } \\
\end{array}$ & $\begin{array}{r}0.98 \\
0.02 \\
0.32 \\
0.64 \\
10 / 23 / 85 \\
\end{array}$ & & $\begin{array}{r}0.93 \\
0.06 \\
0.22 \\
0.65 \\
01 / 17 / 89 \\
\end{array}$ & $\begin{array}{r}0.87 \\
0.08 \\
0.20 \\
0.59 \\
4 / 17 / 90 \\
\end{array}$ \\
\hline $\begin{array}{l}\text { CALORIFIC VALUE (dry) } \\
\text { BTU/Ib } \\
\text { analysis date }\end{array}$ & $\begin{array}{c}14540 \\
10 / 23 / 85\end{array}$ & & & $\begin{array}{c}14392 \\
4 / 17 / 90\end{array}$ \\
\hline $\begin{array}{l}\text { ALKALI EXTRACTION } \\
\% \text { transmission } \\
\text { analysis date }\end{array}$ & & $\begin{array}{c}95.9 \\
06 / 03 / 88\end{array}$ & $\begin{array}{c}97.4 \\
10 / 05 / 88\end{array}$ & $\begin{array}{c}92.1 \\
3 / 02 / 90\end{array}$ \\
\hline
\end{tabular}

note: a) Sample discrepancy with ash analysis. PSU MCL ash, moisture. PSU MCL sulfur forms (revised analysis report). 


\begin{tabular}{|c|c|c|c|c|}
\hline $\begin{array}{l}\text { PSOC-1482 } \\
\text { collection date: } 07\end{array}$ & $\begin{array}{l}\text { HAGEL } \\
07 / 01 / 85\end{array}$ & \multicolumn{3}{|c|}{ ligA, Ro max $=0.39$} \\
\hline $\begin{array}{r}\text { Split ID } \\
\text { slze } \\
\% \text { head oxygen } \\
\text { note } \\
\text { date sealed } \\
\text { date oxygen }\end{array}$ & $\begin{array}{l}\text { analysis } \\
\text { sample } 1 \\
- \text { 60mesh }\end{array}$ & $\begin{array}{l}\text { analysis } \\
\text { sample } 2 \\
-20 \text { mesh }\end{array}$ & $\begin{array}{c}30 \text { gal. } \\
1 \text { of } 3 \\
-1 / 4^{\prime \prime} \\
1.6 \\
a \\
08 / 29 / 85 \\
9 / 23 / 88\end{array}$ & $\begin{array}{l}30 \mathrm{gal} \\
4 \text { of } 4 \\
-1 / 4^{\prime \prime} \\
6.6 \\
9 / 28 / 89\end{array}$ \\
\hline $\begin{array}{l}\text { PROXIMATE } \\
\% \text { molsture } \\
\% \text { ash (dry) } \\
\text { analysis date } \\
\end{array}$ & $\begin{array}{c}34.7 \\
9.0 \\
10 / 24 / 85\end{array}$ & $\begin{array}{r}34.5 \\
8.5 \\
12 / 11 / 86 \\
\end{array}$ & $\begin{array}{c}32.9 \\
7.4 \\
10 / 12 / 88 \\
\end{array}$ & $\begin{array}{c}34.4 \\
9.2 \\
2 / 06 / 90\end{array}$ \\
\hline $\begin{array}{l}\text { SULFUR FORMS (dry \%) } \\
\text { total sulfur } \\
\text { sulfate } \\
\text { pyritic } \\
\text { organic } \\
\text { analysis date }\end{array}$ & $\begin{array}{c}0.86 \\
0.02 \\
0.09 \\
0.75 \\
10 / 24 / 85\end{array}$ & $12 / 11 / 86$ & $\begin{array}{c}0.82 \\
0.14 \\
0.22 \\
0.46 \\
01 / 17 / 89\end{array}$ & $\begin{array}{l}0.88 \\
0.17 \\
0.20 \\
0.51 \\
2 / 06 / 90\end{array}$ \\
\hline $\begin{array}{l}\text { CALORIFIC VALUE (dry) } \\
\text { BTU/lb } \\
\text { analysis date }\end{array}$ & $\begin{array}{c}11118 \\
10 / 24 / 85\end{array}$ & & & $\begin{array}{c}10695 \\
2 / 06 / 90\end{array}$ \\
\hline
\end{tabular}

notes: a) Sample moist, trace yellow \& white sulfates. PSU MCL ash, moisture. PSU MCL sulfur forms (revised analysts report). 


\begin{tabular}{|c|c|c|c|c|}
\hline $\begin{array}{ll}\text { PSOC }-1485 & R C \\
\text { collection date: } & 07\end{array}$ & $\begin{array}{l}\text { ROSEBUD } \\
07 / 05 / 86\end{array}$ & \multicolumn{3}{|c|}{ subB, Ro max $=0.51$} \\
\hline $\begin{array}{r}\text { Split ID } \\
\text { size } \\
\% \text { head oxygen } \\
\text { note } \\
\text { date sealed } \\
\text { date oxygen }\end{array}$ & $\begin{array}{l}\text { analysis } \\
\text { sample } 1 \\
-60 \text { mesh }\end{array}$ & $\begin{array}{l}\# 2 \text { can } \\
15 \text { of } 31 \\
-20 \text { mesh } \\
04 / 01 / 86\end{array}$ & $\begin{array}{c}30 \text { gal } \\
3 \text { of } 3 \\
-1 / 4^{\prime \prime} \\
8.1 \\
a \\
03 / 25 / 86 \\
9 / 26 / 88\end{array}$ & $\begin{array}{l}30 \text { gal. } \\
3 \text { of } 3 \\
-1 / 4^{11} \\
10.4 \\
9 / 29 / 89\end{array}$ \\
\hline $\begin{array}{l}\text { PROXIMATE } \\
\% \text { moisture } \\
\% \text { ash (dry) } \\
\text { analysis date }\end{array}$ & $\begin{array}{c}25.4 \\
11.4 \\
04 / 24 / 86\end{array}$ & $\begin{array}{c}25.0 \\
10.7 \\
06 / 13 / 88\end{array}$ & $\begin{array}{c}21.9 \\
10.4 \\
10 / 12 / 88\end{array}$ & $\begin{array}{c}25.1 \\
11.4 \\
12 / 14 / 89\end{array}$ \\
\hline $\begin{array}{l}\text { SULFUR FORMS (dry \%) } \\
\text { total sulfur } \\
\text { sulfate } \\
\text { pyritic } \\
\text { organic } \\
\text { analysis date }\end{array}$ & $\begin{array}{c}0.88 \\
0.00 \\
0.02 \\
0.86 \\
04 / 24 / 86\end{array}$ & & $\begin{array}{c}0.86 \\
0.08 \\
0.23 \\
0.55 \\
01 / 17 / 89\end{array}$ & $\begin{array}{r}0.77 \\
0.13 \\
0.24 \\
0.40 \\
12 / 14 / 89\end{array}$ \\
\hline $\begin{array}{l}\text { CALORIFIC VALUE (dry) } \\
\text { BTU/lb } \\
\text { analysis date }\end{array}$ & $\begin{array}{c}116 ? 0 \\
04 / 24 / 86\end{array}$ & & & $\begin{array}{c}11449 \\
12 / 14 / 89\end{array}$ \\
\hline $\begin{array}{l}\text { ALKALI EXTRACTION } \\
\% \text { transmission } \\
\text { analysis date }\end{array}$ & & $\begin{array}{c}0.2 \\
06 / 15 / 88\end{array}$ & $\begin{array}{c}0.4 \\
10 / 06 / 88\end{array}$ & $\begin{array}{l}\text { discon- } \\
\text { tinued }\end{array}$ \\
\hline
\end{tabular}

notes: a) Sample moist, good condition. PSU MCL ash, moisture. PSU MCL sulfur forms (revised analysis report). 


\begin{tabular}{|c|c|c|c|c|}
\hline \multirow{2}{*}{$\begin{array}{c}\text { PSOC-1486 } \\
\text { collection date: } \\
\text { Split ID } \\
\text { size } \\
\% \text { head oxygen } \\
\text { note } \\
\text { date sealed } \\
\text { date oxygen }\end{array}$} & $\begin{array}{l}\text { BIG DIRTY } \\
07 / 09 / 85\end{array}$ & \multicolumn{3}{|c|}{ subB, Ro $\max =0.38$} \\
\hline & $\begin{array}{l}\text { analys is } \\
\text { sample } 1 \\
-60 \text { mesh }\end{array}$ & $\begin{array}{l}\# 2 \text { can } \\
17 \text { of } 31 \\
-20 \text { mesh } \\
04 / 02 / 86\end{array}$ & $\begin{array}{c}30 \text { gal } \\
3 \text { of } 3 \\
-1 / 4^{11} \\
4.3 \\
\text { a } \\
03 / 26 / 86 \\
9 / 26 / 88\end{array}$ & $\begin{array}{l}30 \text { gal } \\
3 \text { of } 3 \\
-1 / 4^{11} \\
7.0\end{array}$ \\
\hline $\begin{array}{l}\text { PROXIMATE } \\
\% \text { moisture } \\
\% \text { a sh (dry) } \\
\text { analysis date }\end{array}$ & $\begin{array}{c}20.1 \\
19.4 \\
04 / 24 / 86\end{array}$ & $\begin{array}{c}18.8 \\
18.7 \\
06 / 14 / 88\end{array}$ & $\begin{array}{c}17.3 \\
17.2 \\
10 / 12 / 88\end{array}$ & $\begin{array}{c}19.5 \\
19.3 \\
12 / 14 / 89\end{array}$ \\
\hline $\begin{array}{l}\text { SULFUR FORMS (dry \%) } \\
\text { total sulfur } \\
\text { sulfate } \\
\text { pyritic } \\
\text { organic } \\
\text { analysis date }\end{array}$ & $\begin{array}{c}0.94 \\
0.01 \\
0.25 \\
0.68 \\
04 / 24 / 86\end{array}$ & & $\begin{array}{c}0.91 \\
0.17 \\
0.16 \\
0.58 \\
01 / 17 / 89\end{array}$ & $\begin{array}{c}0.77 \\
0.27 \\
0.12 \\
0.38 \\
12 / 14 / 89\end{array}$ \\
\hline $\begin{array}{l}\text { CALORIFIC VALUE (dry) } \\
\text { BTU/lb } \\
\text { analysis date }\end{array}$ & $\begin{array}{c}10447 \\
04 / 24 / 86\end{array}$ & & & $\begin{array}{c}10267 \\
12 / 14 / 89\end{array}$ \\
\hline $\begin{array}{l}\text { ALKALI EXTRACTION } \\
\% \text { transmission } \\
\text { analysis date }\end{array}$ & & $\begin{array}{c}0.0 \\
06 / 15 / 88\end{array}$ & $\begin{array}{c}0.0 \\
10 / 06 / 88\end{array}$ & $\begin{array}{l}\text { discon- } \\
\text { tinued }\end{array}$ \\
\hline
\end{tabular}

notes: Sample dry, trace yellow sulfate. PSIJ MCL ash, moisture. PSU MCL sulfur forms (revised analysis report). 


\begin{tabular}{|c|c|c|c|c|}
\hline \multirow{2}{*}{\begin{tabular}{|c} 
PSOC-1 187 \\
collection date: \\
Split ID \\
size \\
$\%$ head oxygen \\
note \\
date sealed \\
date oxygen
\end{tabular}} & \multicolumn{4}{|c|}{$\begin{array}{l}\text { ADAVILLE \#1 } \\
07 / 15 / 85\end{array}$} \\
\hline & $\begin{array}{l}\text { analysis } \\
\text { sample } 1 \\
-60 \text { mesh }\end{array}$ & $\begin{array}{l}\# 2 \text { can } \\
15 \text { of } 31 \\
-20 \text { mesh } \\
04 / 03 / 86\end{array}$ & $\begin{array}{c}30 \mathrm{gal} \\
4 \text { of } 4 \\
-1 / 4^{\prime \prime} \\
13.4 \\
\mathrm{a} \\
03 / 26 / 86 \\
10 / 06 / 88\end{array}$ & $\begin{array}{c}30 \text { gal } \\
4 \text { of } 4 \\
-1 / 4^{\prime \prime} \\
17.1 \\
9 / 29 / 89\end{array}$ \\
\hline $\begin{array}{l}\text { PROXIMATE } \\
\% \text { moisture } \\
\% \text { ash (dry) } \\
\text { analysis date }\end{array}$ & $\begin{array}{c}19.0 \\
5.6 \\
04 / 24 / 86 \\
\end{array}$ & $\begin{array}{c}18.1 \\
5.5 \\
06 / 13 / 88 \\
\end{array}$ & $\begin{array}{c}11.5 \\
5.3 \\
11 / 09 / 88 \\
\end{array}$ & $\begin{array}{r}18.3 \\
5.5 \\
12 / 14 / 89 \\
\end{array}$ \\
\hline $\begin{array}{l}\text { SULFUR FORMS (dry \%) } \\
\text { total sulfur } \\
\text { sulfate } \\
\text { pyritic } \\
\text { organic } \\
\text { analysis date }\end{array}$ & $\begin{array}{c}1.31 \\
0.01 \\
0.03 \\
1.27 \\
04 / 24 / 86\end{array}$ & & $\begin{array}{r}1.37 \\
0.18 \\
0.08 \\
1.11 \\
01 / 17 / 89 \\
\end{array}$ & $\begin{array}{r}1.21 \\
0.21 \\
0.06 \\
0.94 \\
12 / 14 / 89 \\
\end{array}$ \\
\hline $\begin{array}{l}\text { CALORIFIC VALUE (dry) } \\
\text { BTU/Ib } \\
\text { analysis date }\end{array}$ & $\begin{array}{c}12589 \\
04 / 24 / 86\end{array}$ & & & $\begin{array}{c}12414 \\
12 / 14 / 89\end{array}$ \\
\hline $\begin{array}{l}\text { ALKALI EXTRACTION } \\
\% \text { transmission } \\
\text { analysis date }\end{array}$ & & $\begin{array}{c}1.7 \\
06 / 15 / 88\end{array}$ & $\begin{array}{l}\text { discon- } \\
\text { tinued }\end{array}$ & \\
\hline
\end{tabular}

notes: Sample dry, trace yellow sulfate. PSU MCL sulfur forms (revised analysis report). 


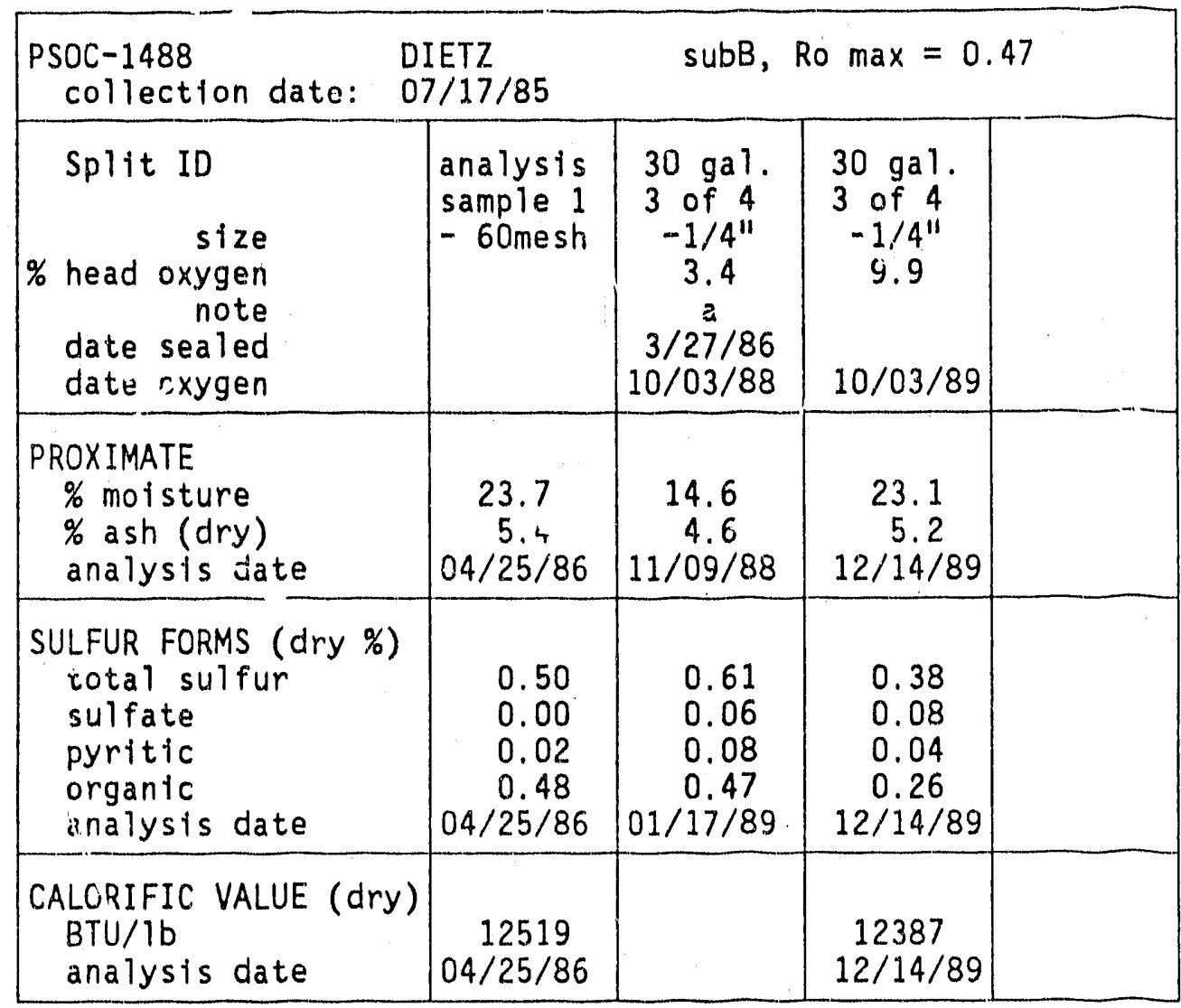

notes: a) Sample moist, good condition. PSU MCL sulfur forms (revised analysis report). 


\begin{tabular}{|c|c|c|c|c|}
\hline $\begin{array}{ll}\text { PSOC }-1489 & P U \\
\text { collection date: } & 07\end{array}$ & $\begin{array}{l}\text { PUST } \\
07 / 19 / 85\end{array}$ & \multicolumn{3}{|c|}{ ligA, Ro $\max =0.34$} \\
\hline $\begin{array}{r}\text { Split ID } \\
\text { size } \\
\% \text { head oxygen } \\
\text { note } \\
\text { date sealed } \\
\text { date oxygen }\end{array}$ & $\begin{array}{l}\text { analysis } \\
\text { sample } 1 \\
-60 \text { mesh }\end{array}$ & $\begin{array}{c}30 \text { gal. } \\
4 \text { of } 4 \\
-1 / 4^{\prime \prime} \\
10.6 \\
a \\
10 / 06 / 88\end{array}$ & $\begin{array}{l}30 \text { gal. } \\
4 \text { of } 4 \\
-1 / 4^{\prime \prime} \\
14.3 \\
9 / 29 / 89\end{array}$ & \\
\hline $\begin{array}{l}\text { PROXIMATE } \\
\% \text { molsture } \\
\% \text { ash (dry) } \\
\text { analysis date }\end{array}$ & $\begin{array}{c}37.2 \\
11.0 \\
04 / 25 / 86\end{array}$ & $\begin{array}{c}20.1 \\
10.5 \\
11 / 09 / 88\end{array}$ & $\begin{array}{r}34.8 \\
10.9 \\
12 / 14 / 89 \\
\end{array}$ & \\
\hline $\begin{array}{l}\text { SULFUR FORMS (dry \%) } \\
\text { total sulfur } \\
\text { sulfate } \\
\text { pyritic } \\
\text { organic } \\
\text { analysis date } \\
\end{array}$ & $\begin{array}{c}0.77 \\
0.00 \\
0.02 \\
0.75 \\
04 / 25 / 86 \\
\end{array}$ & $\begin{array}{r}0.77 \\
0.26 \\
0.25 \\
0.26 \\
01 / 17 / 89 \\
\end{array}$ & $\begin{array}{r}0.52 \\
0.32 \\
0.15 \\
0.04 \\
12 / 14 / 89 \\
\end{array}$ & \\
\hline $\begin{array}{l}\text { CALORIFIC VALUE (dry) } \\
\text { BTU/lb } \\
\text { analysis date }\end{array}$ & $\begin{array}{c}10668 \\
04 / 25 / 86 \\
\end{array}$ & & $\begin{array}{c}10515 \\
12 / 14 / 89 \\
\end{array}$ & \\
\hline
\end{tabular}

notes: a) Sample moist, trace yellow and white sulfate. PSU MCL silfur forms (revised analysis report). 


\begin{tabular}{|c|c|c|c|c|c|}
\hline \multirow{2}{*}{\begin{tabular}{|c}
$\begin{array}{r}\text { PSOC-1490 } \\
\text { collection date: }\end{array}$ \\
Split ID \\
size \\
$\%$ head oxygen \\
note \\
date sealed \\
date oxygen
\end{tabular}} & \multicolumn{5}{|c|}{$\begin{array}{l}\text { BRAZIL BLOCK } \\
7 / 2985\end{array}$} \\
\hline & $\begin{array}{l}\text { analysis } \\
\text { sample } 1 \\
-60 \text { mesh }\end{array}$ & $\begin{array}{l}\text { analysis } \\
\text { sample } 2 \\
-60 \text { mesh }\end{array}$ & $\begin{array}{l}\# 2 \text { can } \\
15 \text { of } 24 \\
-20 \text { mesh } \\
10 / 30 / 85\end{array}$ & $\begin{array}{c}30 \text { ga } 1 \\
3 \text { of } 3 \\
-1 / 4^{\prime \prime} \\
16.4 \\
a \\
10 / 22 / 85 \\
10 / 06 / 88\end{array}$ & $\begin{array}{l}30 \text { gal. } \\
3 \text { of } 3 \\
-1 / 4^{\prime \prime} \\
16.8 \\
9 / 29 / 89\end{array}$ \\
\hline $\begin{array}{l}\text { PROXIMATE } \\
\% \text { moisture } \\
\% \text { ash (dry) } \\
\text { analysis date }\end{array}$ & $\begin{array}{c}15.1 \\
5.4 \\
12 / 23 / 85\end{array}$ & $\begin{array}{c}15.2 \\
5.3 \\
05 / 30 / 86\end{array}$ & $\begin{array}{c}15.5 \\
5.2 \\
06 / 14 / 88\end{array}$ & $\begin{array}{c}9.1 \\
5.2 \\
11 / 09 / 88\end{array}$ & $\begin{array}{r}16.5 \\
5.3 \\
12 / 14 / 89\end{array}$ \\
\hline $\begin{array}{l}\text { GIESELER FLUIDITY } \\
\text { max. ddpm } \\
\text { fluid range } \\
\text { analysis date }\end{array}$ & $\begin{array}{l}0 \\
0\end{array}$ & & & & \\
\hline $\begin{array}{l}\text { SULFUR FORMS } \\
\text { total sulfur } \\
\text { sulfate } \\
\text { pyritic } \\
\text { organic } \\
\text { analysis date }\end{array}$ & $\begin{array}{c}0.71 \\
0.01 \\
0.09 \\
0.61 \\
12 / 23 / 85\end{array}$ & $05 / 30 / 86$ & & $\begin{array}{c}0.76 \\
0.03 \\
0.11 \\
0.62 \\
01 / 17 / 89\end{array}$ & $\begin{array}{c}0.67 \\
0.10 \\
0.06 \\
0.51 \\
12 / 14 / 89\end{array}$ \\
\hline $\begin{array}{l}\text { CALORIFIC VALUE (dry) } \\
\text { BTU/Ib } \\
\text { analysis date }\end{array}$ & $\begin{array}{c}13960 \\
12 / 23 / 85\end{array}$ & $\begin{array}{c}13966 \\
05 / 30 / 86\end{array}$ & & & $\begin{array}{c}13692 \\
12 / 14 / 89\end{array}$ \\
\hline $\begin{array}{c}\text { ALKALI EXTRACTION } \\
\% \text { transmission } \\
\text { analysis date }\end{array}$ & & & $\begin{array}{c}95.9 \\
06 / 15 / 88\end{array}$ & & $\begin{array}{r}77.8 \\
1 / 10 / 90\end{array}$ \\
\hline
\end{tabular}

notes: a) Sample moist, good condition. PSU MCL sulfur forms (revised analysis report). 


\begin{tabular}{|c|c|c|c|c|c|}
\hline $\begin{array}{l}\text { PSOC-14E1 } \\
\text { collection date: }\end{array}$ & $\begin{array}{l}\text { NDIANA \# } \\
/ 29 / 85\end{array}$ & hrCb, Ro & $\max =0$ & & \\
\hline$\left\{\begin{array}{r}\text { Split ID } \\
\text { size } \\
\text { \% head oxygen } \\
\text { note } \\
\text { date sealed } \\
\text { date oxygen }\end{array}\right.$ & $\begin{array}{l}\text { analysis } \\
\text { sample 1 } \\
\text { - 60mesh }\end{array}$ & $\begin{array}{l}\text { analysis } \\
\text { sample } 2\end{array}$ & $\begin{array}{l}\# 2 \text { can } \\
10 \text { of } 29 \\
-20 \text { mesh } \\
10 / 30 / 85\end{array}$ & $\begin{array}{c}30 \text { ga } 1 \\
1 \text { of } 3 \\
-1 / 4^{\prime \prime} \\
15.0 \\
\text { a } \\
10 / 14 / 88\end{array}$ & $\begin{array}{l}30 \text { gal. } \\
1 \text { of } 3 \\
-1 / 4^{\prime \prime} \\
16.4 \\
10 / 03 / 89\end{array}$ \\
\hline $\begin{array}{l}\text { PROXIMATE } \\
\% \text { moisture (a,d) } \\
\% \text { ash (dry) } \\
\text { analysis date }\end{array}$ & $\begin{array}{c}11.5 \\
15.8 \\
05 / 28 / 86\end{array}$ & $\begin{array}{c}11.5 \\
15.2 \\
05 / 30 / 86\end{array}$ & $\begin{array}{c}9.9 \\
14.2 \\
3 / 10 / 88\end{array}$ & $\begin{array}{c}9.6 \\
14.9 \\
02 / 01 / 89\end{array}$ & $\begin{array}{r}11.3 \\
15.2 \\
12 / 14 / 89\end{array}$ \\
\hline $\begin{array}{l}\text { GIESELER FLUIDITY } \\
\text { max. ddpm } \\
\text { fluid range } \\
\text { analysis date }\end{array}$ & $\begin{array}{r}28 \\
61 \\
11 / 27 / 85\end{array}$ & & $\begin{array}{c}0 \\
0 \\
3 / 21 / 88\end{array}$ & $\begin{array}{c}0 \\
0 \\
11 / 21 / 88\end{array}$ & $\begin{array}{l}\text { discon- } \\
\text { tinued }\end{array}$ \\
\hline $\begin{array}{l}\text { SULFUR FORMS (dry) } \\
\text { total sulfur } \\
\text { sulfate } \\
\text { pyritic } \\
\text { organic } \\
\text { analysis date }\end{array}$ & $\begin{array}{c}2.82 \\
0.02 \\
2.22 \\
0.57 \\
12 / 23 / 85 \\
\end{array}$ & $05 / 30 / 86$ & & & $\begin{array}{r}2.56 \\
1.30 \\
0.60 \\
0.66 \\
12 / 1.4 / 89\end{array}$ \\
\hline $\begin{array}{l}\text { CALORIFIC VALUE (dry) } \\
\text { BTU/Ib } \\
\text { analysis date }\end{array}$ & $\begin{array}{c}12134 \\
12 / 23 / 85\end{array}$ & $\begin{array}{c}12292 \\
05 / 30 / 86\end{array}$ & & & $\begin{array}{c}11613 \\
12 / 14 / 89\end{array}$ \\
\hline $\begin{array}{l}\text { ALKALI EXTRACTION } \\
\% \text { transinission } \\
\text { analysis date }\end{array}$ & & & $\begin{array}{c}95.1 \\
06 / 07 / 88\end{array}$ & $\begin{array}{c}93.6 \\
12 / 09 / 88\end{array}$ & $\begin{array}{l}97.3 \\
1 / 08 / 90\end{array}$ \\
\hline
\end{tabular}

notes: a) Sample moist, good condition. PSU MCL ash, moisture; s forms (revised) 


\begin{tabular}{|c|c|c|c|c|c|}
\hline $\begin{array}{ll}\text { PSOC- } 1492 & \text { IL } \\
\text { collection date: } & \text { O\& }\end{array}$ & $\begin{array}{l}\text { LLINOIS \#! } \\
8 / 01 / 85\end{array}$ & $h \vee C b, R$ & $0 \max =0$. & & \\
\hline $\begin{array}{r}\text { split ID } \\
\text { size } \\
\text { \% head oxygen } \\
\text { note } \\
\text { date sealed } \\
\text { date oxygen }\end{array}$ & $\begin{array}{l}\text { analysis } \\
\text { sample } 1 \\
-60 \text { mesh }\end{array}$ & $\begin{array}{l}\text { analysis } \\
\text { sample } 2\end{array}$ & $\begin{array}{l}\# 2 \text { can } \\
31 \text { of } 31 \\
-20 \text { mesh } \\
11 / 14 / 85\end{array}$ & $\begin{array}{c}30 \text { gal. } \\
1 \text { of } 3 \\
-1 / 4^{\prime \prime} \\
16.1 \\
a \\
10 / 14 / 88\end{array}$ & $\begin{array}{l}30 \mathrm{gal} \\
1 \text { of } 3 \\
-1 / 4^{\prime \prime} \\
18.3 \\
10 / 03 / 89\end{array}$ \\
\hline $\begin{array}{l}\text { PROXIMATE } \\
\% \text { moisture (a.d) } \\
\% \text { a si (dry) } \\
\text { analysis date }\end{array}$ & $\begin{array}{c}9.6 \\
10.6 \\
12 / 23 / 85\end{array}$ & $\begin{array}{c}9.5 \\
10.5 \\
05 / 29 / 86\end{array}$ & $\begin{array}{c}9.2 \\
9.9 \\
03 / 14 / 88\end{array}$ & $\begin{array}{c}8.0 \\
10.2 \\
02 / 01 / 89\end{array}$ & $\begin{array}{c}9.8 \\
10.5 \\
02 / 06 / 90\end{array}$ \\
\hline $\begin{array}{l}\text { GIESELER FLUIDITY } \\
\text { max. ddpm } \\
\text { fluid range } \\
\text { analysis date }\end{array}$ & $\begin{array}{r}6 \\
56 \\
11 / 29 / 85\end{array}$ & & $\begin{array}{r}5 \\
58 \\
03 / 14 / 88\end{array}$ & $\begin{array}{c}0 \\
0 \\
11 / 21 / 88\end{array}$ & \\
\hline $\begin{array}{l}\text { SULFUR FORMS (dry) } \\
\text { total sulfur }(3.90) \\
\text { sulfate } \quad(0.03) \\
\text { pyritic } \quad(1.82) \\
\text { organic } \quad(2.05) \\
\text { analysis date. }\end{array}$ & $\begin{array}{r}3.90 \\
0.03 \\
1.82 \\
2.05 \\
12 / 2.3 / 85 \\
\end{array}$ & $\begin{array}{r}4.35 \\
05 / 29 / 86\end{array}$ & & $\begin{array}{r}4.10 \\
0.91 \\
0.76 \\
2.43 \\
2 / 12 / 90\end{array}$ & $\begin{array}{r}3.90 \\
0.94 \\
0.62 \\
2.34 \\
02 / 06 / 90\end{array}$ \\
\hline $\begin{array}{l}\text { CALORIFIC VALUE (dry) } \\
\text { BTU/Ib } \\
\text { analysis date }\end{array}$ & $\begin{array}{c}12758 \\
12 / 23 / 85 \\
\end{array}$ & $\begin{array}{c}12722 \\
05 / 29 / 86\end{array}$ & & & $\begin{array}{c}12237 \\
02 / 06 / 90\end{array}$ \\
\hline $\begin{array}{l}\text { ALKALI EXTRACTION } \\
\% \text { transinission } \\
\text { analysis date }\end{array}$ & & & $\begin{array}{c}86.9 \\
06 / 07 / 88\end{array}$ & $\begin{array}{c}93.4 \\
12 / 09 / 88\end{array}$ & $\begin{array}{c}86.2 \\
01 / 11 / 90\end{array}$ \\
\hline
\end{tabular}

notes: a) Sample dry, good condition. PSU MCL ash, moisture; $S$ forms (revised). 


\begin{tabular}{|c|c|c|c|c|c|}
\hline $\begin{array}{l}\text { PSOC-1493 } \\
\quad \text { collection date: }\end{array}$ & $\begin{array}{l}\text { LLINOIS \#6 } \\
18 / 01 / 85\end{array}$ & huCb, & $0 \max =0$ & & \\
\hline $\begin{array}{r}\text { split ID } \\
\text { size } \\
\% \text { head oxygen } \\
\text { note } \\
\text { date sealed } \\
\text { date oxygen }\end{array}$ & $\begin{array}{l}\text { analysis } \\
\text { sample } 1 \\
-60 \text { mesh }\end{array}$ & $\begin{array}{l}\text { analysis } \\
\text { sample } 2\end{array}$ & $\begin{array}{l}\# 2 \text { can } \\
19 \text { of } 31 \\
-20 \text { mesh } \\
11 / 19 / 85\end{array}$ & $\begin{array}{c}30 \mathrm{gal} \\
2 . \text { of } 4 \\
-1 / 4^{11} \\
3.0 \\
a \\
10 / 14 / 88\end{array}$ & $\begin{array}{l}30 \text { gal } \\
2 \text { of } 4 \\
-1 / 4^{\prime \prime} \\
15.4 \\
10 / 03 / 89\end{array}$ \\
\hline $\begin{array}{l}\text { PROXIMATE } \\
\% \text { moisture } \\
\% \text { ash (dry) } \\
\text { analysis date }\end{array}$ & $\begin{array}{c}9.5 \\
15.2 \\
12 / 23 / 85 \\
\end{array}$ & $\begin{array}{c}9.4 \\
15.2 \\
05 / 29 / 86\end{array}$ & $\begin{array}{c}9.8 \\
14.3 \\
03 / 17 / 88\end{array}$ & $\begin{array}{c}8.8 \\
14.6 \\
02 / 01 / 89\end{array}$ & $\begin{array}{c}10.2 \\
14.4 \\
02 / 06 / 90\end{array}$ \\
\hline $\begin{array}{l}\text { GIESELER FLUIDITY } \\
\text { max. ddpm } \\
\text { fluid range } \\
\text { analysis date }\end{array}$ & $\begin{array}{r}6 \\
47 \\
12 / 05 / 85\end{array}$ & & $\begin{array}{c}6 \\
51 \\
03 / 17 / 88\end{array}$ & $\begin{array}{c}0 \\
0 \\
11 / 23 / 88\end{array}$ & $\begin{array}{l}\text { discon- } \\
\text { tinued }\end{array}$ \\
\hline $\begin{array}{l}\text { SULFUR FORMS (dry) } \\
\text { total sulfur } \\
\text { sulfate } \\
\text { pyritic } \\
\text { organic } \\
\text { analysis date }\end{array}$ & $\begin{array}{c}4.23 \\
0.04 \\
2.61 \\
1.57 \\
12 / 23 / 85 \\
\end{array}$ & $\begin{array}{r}4.98 \\
05 / 29 / 86 \\
\end{array}$ & & $\begin{array}{l}4.51 \\
0.68 \\
1.40 \\
2.43 \\
02 / 12 / 90\end{array}$ & $\begin{array}{r}4.07 \\
1.04 \\
1.02 \\
2.01 \\
02 / 06 / 90\end{array}$ \\
\hline $\begin{array}{l}\text { CALORIFIC VALUE (dry) } \\
\text { BTU/Ib } \\
\text { analysis date }\end{array}$ & $\begin{array}{c}11989 \\
12 / 23 / 85\end{array}$ & $\begin{array}{c}11963 \\
05 / 29 / 86\end{array}$ & & & $\begin{array}{c}11746 \\
02 / 06 / 90\end{array}$ \\
\hline $\begin{array}{l}\text { ALKALI EXTRACTION } \\
\% \text { transmission } \\
\text { analysis date }\end{array}$ & & & $\begin{array}{c}93.2 \\
06 / 08 / 88\end{array}$ & $\begin{array}{c}94.5 \\
12 / 12 / 88\end{array}$ & $\begin{array}{c}89.3 \\
01 / 09 / 90\end{array}$ \\
\hline
\end{tabular}

notes: a) Sample molst, trace yellow sulfate. PSU MCL ash, molsture; $S$ forms (revised). 


\begin{tabular}{|c|c|c|c|c|}
\hline \multirow{2}{*}{$\begin{array}{l}\text { PSOC-1494 } \\
\text { collection date: } \\
\text { split ID } \\
\text { size } \\
\text { \% head oxygen } \\
\text { note } \\
\text { date sealed } \\
\text { date oxygen }\end{array}$} & \multicolumn{4}{|c|}{$\begin{array}{l}\text { KENTUCKY \#9 hvBb, Ro } \max =0.61 \\
08 / 02 / 85\end{array}$} \\
\hline & $\begin{array}{l}\text { analysis } \\
\text { sample } 1 \\
-60 \text { mesh }\end{array}$ & $\begin{array}{l}\# 2 \text { can } \\
8 \text { of } 31 \\
-20 \text { mesh } \\
11 / 19 / 85\end{array}$ & $\begin{array}{c}30 \text { gal. } \\
1 \text { of } 3 \\
-1 / 4^{\prime \prime} \\
13.7 \\
\text { a } \\
10 / 14 / 88\end{array}$ & $\begin{array}{l}30 \text { gal. } \\
1 \text { of } 3 \\
-1 / 4^{\prime \prime} \\
18.1 \\
10 / 03 / 89\end{array}$ \\
\hline $\begin{array}{l}\text { PROXIMATE } \\
\% \text { moisture (a.d) } \\
\% \text { ash (dry) } \\
\text { analysis date }\end{array}$ & $\begin{array}{c}7.1 \\
11.8 \\
12 / 23 / 85\end{array}$ & $\begin{array}{c}7.1 \\
11.4 \\
03 / 21 / 88\end{array}$ & $\begin{array}{c}6.7 \\
10.7 \\
02 / 01 / 89\end{array}$ & $\begin{array}{c}8.1 \\
11.4 \\
02 / 06 / 90\end{array}$ \\
\hline $\begin{array}{l}\text { GIESELER FLUIDITY } \\
\text { max. ddpm } \\
\text { fluid range } \\
\text { analysis date }\end{array}$ & $\begin{array}{r}48 \\
60 \\
12 / 03 / 85\end{array}$ & $\begin{array}{c}175 \\
84 \\
03 / 21 / 88\end{array}$ & $\begin{array}{c}0 \\
0 \\
11 / 30 / 88\end{array}$ & \\
\hline $\begin{array}{l}\text { SULFUR FORMS (dry) } \\
\text { total sulfur } \\
\text { sulfate } \\
\text { pyritic } \\
\text { organic } \\
\text { analysis date }\end{array}$ & $\begin{array}{r}4.85 \\
0.02 \\
3.14 \\
1.69 \\
12 / 23 / 85 \\
\end{array}$ & & $\begin{array}{c}5.45 \\
1.21 \\
1.63 \\
2.61 \\
02 / 12 / 90 \\
\end{array}$ & $\begin{array}{r}5.15 \\
1.21 \\
1.75 \\
2.19 \\
02 / 06 / 90 \\
\end{array}$ \\
\hline $\begin{array}{l}\text { CALORIFIC VALUE (dry) } \\
\text { BTU/lb } \\
\text { analysis date }\end{array}$ & $\begin{array}{c}12851 \\
12 / 23 / 85\end{array}$ & & & $\begin{array}{r}12287 \\
02 / 06 / 90 \\
\end{array}$ \\
\hline $\begin{array}{c}\text { ALKALI EXTRACTION } \\
\% \text { transmission } \\
\text { analysis date }\end{array}$ & & $\begin{array}{c}98.4 \\
06 / 08 / 88\end{array}$ & $\begin{array}{c}94.7 \\
12 / 12 / 88\end{array}$ & $\begin{array}{c}94.8 \\
01 / 11 / 90\end{array}$ \\
\hline
\end{tabular}

notes: a) Sample dry, fron stained. PSU MCL ash, moisture; S forms (revised). 


\begin{tabular}{|c|c|c|c|c|c|}
\hline \multirow{2}{*}{$\begin{array}{l}\text { PSOC-1495 } \\
\text { collection date: } \\
\text { split ID } \\
\text { size } \\
\% \text { head oxygen } \\
\text { note } \\
\text { date sealed } \\
\text { date oxygen }\end{array}$} & \multicolumn{5}{|c|}{$\begin{array}{l}\text { BEVIER-WHEELER hvCb, Ro } \max =0.62 \\
08 / 05 / 85\end{array}$} \\
\hline & $\begin{array}{l}\text { analysis } \\
\text { sample } 1 \\
-60 \text { mesh }\end{array}$ & $\begin{array}{l}\text { analysis } \\
\text { sample } 2 \\
-60 \text { mesh }\end{array}$ & $\begin{array}{l}\# 2 \text { can } \\
23 \text { of } 31 \\
-20 \text { mesh } \\
11 / 21 / 85\end{array}$ & $\begin{array}{c}30 \text { gal } \\
3 \text { of } 3 \\
-1^{\prime \prime} \\
15.8 \\
a \\
10 / 14 / 88\end{array}$ & $\begin{array}{l}30 \text { gal } \\
1 \text { of } 3 \\
-1 / f^{\prime \prime} \\
17.9 \\
10 / 11 / 89\end{array}$ \\
\hline $\begin{array}{l}\text { PROXIMATE } \\
\% \text { moisture (a.d) } \\
\% \text { ash (dry) } \\
\text { analysis date }\end{array}$ & $\begin{array}{c}13.4 \\
16.1 \\
12 / 30 / 85 \\
\end{array}$ & $\begin{array}{r}13.3 \\
16.1 \\
05 / 30 / 86 \\
\end{array}$ & $\begin{array}{r}10.2 \\
14.9 \\
03 / 22 / 88 \\
\end{array}$ & $\begin{array}{c}11.0 \\
15.1 \\
02 / 01 / 89 \\
\end{array}$ & $\begin{array}{r}13.0 \\
15.8 \\
02 / 06 / 90 \\
\end{array}$ \\
\hline $\begin{array}{l}\text { GIESELER FLUIDITY } \\
\text { max. ddpm } \\
\text { fluid range } \\
\text { analysis date }\end{array}$ & $\begin{array}{r}2 \\
45 \\
12 / 04 / 85\end{array}$ & & $\begin{array}{c}0 \\
0 \\
03 / 22 / 88 \\
\end{array}$ & $\begin{array}{c}0 \\
0 \\
12 / 02 / 88 \\
\end{array}$ & $\begin{array}{l}\text { discon- } \\
\text { tinued }\end{array}$ \\
\hline $\begin{array}{l}\text { SULFUR FORMS (dry) } \\
\text { total sulfur } \\
\text { sulfate } \\
\text { pyritic } \\
\text { organic } \\
\text { analysis date }\end{array}$ & $\begin{array}{c}6.04 \\
0.03 \\
3.77 \\
2.24 \\
12 / 30 / 85 \\
\end{array}$ & $\begin{array}{c}7.02 \\
0.22 \\
3.43 \\
3.37 \\
06 / 18 / 86 \\
\end{array}$ & & $\begin{array}{r}6.47 \\
1.89 \\
1.49 \\
3.09 \\
02 / 12 / 90 \\
\end{array}$ & $\begin{array}{r}6.05 \\
1.97 \\
1.59 \\
2.49 \\
02 / 06 / 90 \\
\end{array}$ \\
\hline $\begin{array}{l}\text { CALORIFIC VALUE (dry) } \\
\text { BTU/1b } \\
\text { analysis date }\end{array}$ & $\begin{array}{c}14218 \\
12 / 20 / 85\end{array}$ & $\begin{array}{c}14143 \\
05 / 30 / 86\end{array}$ & & & $\begin{array}{c}11085 \\
02 / 06 / 90\end{array}$ \\
\hline $\begin{array}{l}\text { ALKALI EXTRACTION } \\
\% \text { transmission } \\
\text { analysis date }\end{array}$ & & & $\begin{array}{c}87.4 \\
06 / 07 / 88\end{array}$ & $\begin{array}{c}93.0 \\
12 / 12 / 88\end{array}$ & $\begin{array}{c}94.6 \\
01 / 12 / 90\end{array}$ \\
\hline
\end{tabular}

notes: a) PSU MCL ash, moisture; $S$ forms (revised). 


\begin{tabular}{|c|c|c|c|c|}
\hline $\begin{array}{l}\text { PSOC }-1496 \\
\text { collection date: }\end{array}$ & $\begin{array}{l}\text { EBO } \\
18 / 05 / 85\end{array}$ & hvCb, & Ro $\max =$ & \\
\hline $\begin{array}{r}\text { Split ID } \\
\text { size } \\
\% \text { head oxygen } \\
\text { note } \\
\text { date sealed } \\
\text { date oxygen }\end{array}$ & $\begin{array}{l}\text { analysis } \\
\text { sample } 1 \\
-60 \text { mesh }\end{array}$ & $\begin{array}{l}\# 2 \text { Can } \\
13 \text { of } 30 \\
-20 \text { mesh } \\
11 / 27 / 85\end{array}$ & $\begin{array}{c}30 \text { gal } \\
3 \text { of } 3 \\
-1 / 4^{11} \\
12.8 \\
a \\
10 / 14 / 88\end{array}$ & $\begin{array}{l}30 \text { gal } \\
3 \text { of } 3 \\
-1 / 4^{11} \\
19.0 \\
10 / 11 / 89\end{array}$ \\
\hline $\begin{array}{l}\text { PROXIMATE } \\
\% \text { moisture (a.d) } \\
\% \text { ash (dry) } \\
\text { analysis date }\end{array}$ & $\begin{array}{c}7.9 \\
29.4 \\
12 / 23 / 85\end{array}$ & $\begin{array}{c}7.3 \\
29.5 \\
03 / 28 / 88\end{array}$ & $\begin{array}{c}6.3 \\
27.4 \\
02 / 01 / 89\end{array}$ & $\begin{array}{c}8.3 \\
28.5 \\
02 / 06 / 90\end{array}$ \\
\hline $\begin{array}{l}\text { GIESELER FLUIDITY } \\
\text { max. ddpm } \\
\text { fluid range } \\
\text { analysis date }\end{array}$ & $\begin{array}{r}6 \\
44 \\
12 / 04 / 85\end{array}$ & $\begin{array}{c}6 \\
39 \\
03 / 28 / 88\end{array}$ & $\begin{array}{c}0 \\
0 \\
12 / 02 / 88\end{array}$ & $\begin{array}{l}\text { discon- } \\
\text { tinued }\end{array}$ \\
\hline $\begin{array}{l}\text { SULFUR FORMS (dry \%) } \\
\text { total sulfur } \\
\text { sulfate } \\
\text { pyrittc } \\
\text { organtc } \\
\text { analysis date }\end{array}$ & $\begin{array}{c}6.04 \\
0.03 \\
3.31 \\
2.69 \\
12 / 23 / 85\end{array}$ & & $\begin{array}{l}6.23 \\
2.09 \\
1.75 \\
2.39 \\
02 / 12 / 90\end{array}$ & $\begin{array}{r}5.74 \\
2.02 \\
1.93 \\
1.79 \\
02 / 06 / 90\end{array}$ \\
\hline $\begin{array}{l}\text { CALORIFIC VALUE (dry) } \\
\text { BTU/1b } \\
\text { analysis date }\end{array}$ & $\begin{array}{c}9881 \\
12 / 23 / 85\end{array}$ & & & $\begin{array}{c}9619 \\
02 / 06 / 90\end{array}$ \\
\hline $\begin{array}{c}\text { ALKALI EXTRACTION } \\
\% \text { transmission } \\
\text { analysis date }\end{array}$ & & $\begin{array}{c}94,4 \\
06 / 30 / 88\end{array}$ & $\begin{array}{c}94.7 \\
12 / 12 / 88\end{array}$ & $\begin{array}{c}80.9 \\
01 / 12 / 90\end{array}$ \\
\hline
\end{tabular}

notes: a) Sample dry, trace white sulfate. PSU MCL ash, moisture (low ash may be due to lab not sample split); $S$ forms (revised). 


\begin{tabular}{|c|c|c|c|c|c|}
\hline $\begin{aligned} & \text { PSOC }-1497 \text { I } \\
& \text { collection date: } 0 \\
&\end{aligned}$ & $\begin{array}{l}\text { LINOIS \#2 } \\
8 / 08 / 85\end{array}$ & hvCb, & Ro $\max =$ & 40 & \\
\hline $\begin{array}{r}\text { Split ID } \\
\text { size } \\
\% \text { head oxygen } \\
\text { note } \\
\text { date sealed } \\
\text { date oxygen }\end{array}$ & $\begin{array}{l}\text { analysis } \\
\text { sample } 1 \\
-60 \text { mesh }\end{array}$ & $\begin{array}{l}\text { analysis } \\
\text { sample } 2\end{array}$ & $\begin{array}{l}\# 2 \text { can } \\
13 \text { of } 30 \\
-20 \text { mesh } \\
12 / 03 / 85\end{array}$ & $\begin{array}{c}30 \text { gal. } \\
1 \text { of } 3 \\
-1 / 4^{11} \\
11.9 \\
a \\
10 / 14 / 88\end{array}$ & $\begin{array}{l}30 \text { gal. } \\
1 \text { of } 3 \\
-1 / 4^{\prime \prime} \\
16.0 \\
10 / 04 / 89\end{array}$ \\
\hline $\begin{array}{l}\text { PROXIMATE } \\
\% \text { moisture (a.d) } \\
\% \text { ash (dry) } \\
\text { analysis date }\end{array}$ & $\begin{array}{c}13.5 \\
12.0 \\
12 / 23 / 85\end{array}$ & $\begin{array}{c}13.4 \\
11.7 \\
05 / 30 / 86\end{array}$ & $\begin{array}{c}14.6 \\
12.3 \\
03 / 29 / 88\end{array}$ & $\begin{array}{c}12.1 \\
10.8 \\
02 / 01 / 88\end{array}$ & $\begin{array}{c}13.2 \\
11.5 \\
02 / 06 / 90\end{array}$ \\
\hline $\begin{array}{l}\text { GIESELER FLUIDITY } \\
\text { max. ddpm } \\
\text { fluid range } \\
\text { analysis date }\end{array}$ & $\begin{array}{r}83 \\
85 \\
12 / 06 / 85\end{array}$ & & $\begin{array}{c}146 \\
87 \\
03 / 29 / 87\end{array}$ & $\begin{array}{c}0 \\
0 \\
12 / 05 / 88\end{array}$ & $\begin{array}{l}\text { discon- } \\
\text { tinued }\end{array}$ \\
\hline $\begin{array}{l}\text { SULFUR FORMS (dry \%) } \\
\text { total sulfur } \\
\text { sulfate } \\
\text { pyritic } \\
\text { organic } \\
\text { analysis date }\end{array}$ & $\begin{array}{c}5.04 \\
0.02 \\
4.00 \\
1.02 \\
12 / 23 / 85\end{array}$ & $\begin{array}{c}5.93 \\
0.35 \\
3.51 \\
2.06 \\
06 / 18 / 86\end{array}$ & & $\begin{array}{c}5.33 \\
2.20 \\
1.21 \\
1.92 \\
02 / 12 / 90\end{array}$ & $\begin{array}{r}4.96 \\
2.16 \\
1.43 \\
1.37 \\
02 / 06 / 90\end{array}$ \\
\hline $\begin{array}{l}\text { CALORIFIC VALUE (dry) } \\
\text { BTU/lb } \\
\text { analysis date }\end{array}$ & $\begin{array}{c}12823 \\
12 / 23 / 85\end{array}$ & $\begin{array}{c}12778 \\
05 / 30 / 86\end{array}$ & & & $\begin{array}{c}11748 \\
02 / 06 / 90\end{array}$ \\
\hline $\begin{array}{l}\text { ALKALI EXTRACTION } \\
\% \text { transmission } \\
\text { analysis date }\end{array}$ & & & $\begin{array}{c}96.3 \\
06 / 08 / 88\end{array}$ & $\begin{array}{c}93.5 \\
12 / 14 / 88\end{array}$ & $\begin{array}{c}88.9 \\
01 / 23 / 90\end{array}$ \\
\hline
\end{tabular}

notes: a) Sample dry, visible white sulfate. PSU MCL ash, moisture; $S$ forms (revised). 


\begin{tabular}{|c|c|c|c|c|}
\hline $\begin{array}{l}\text { PSOC-1498 } \\
\text { collection date: } \\
\end{array}$ & $\begin{array}{l}\text { WADGE } \\
09 / 24 / 85\end{array}$ & \multicolumn{3}{|c|}{ hvCb, Ro $\max =0.60$} \\
\hline $\begin{array}{r}\text { Split ID } \\
\text { size } \\
\% \text { head oxygen } \\
\text { note } \\
\text { date sealed } \\
\text { date oxygen }\end{array}$ & $\begin{array}{l}\text { analysis } \\
\text { sample } 1 \\
-60 \text { mesh }\end{array}$ & $\begin{array}{l}\# 2 \text { Can } \\
15 \text { of } 31 \\
-20 \text { mesh } \\
12 / 13 / 85\end{array}$ & $\begin{array}{c}30 \mathrm{gal} \\
3 \text { of } 3 \\
-1 / 4^{11} \\
15.6 \\
\mathrm{a} \\
10 / 14 / 88\end{array}$ & $\begin{array}{l}30 \text { gal. } \\
3 \text { of } 3 \\
-1 / 4^{\prime \prime} \\
17.1 \\
10 / 04 / 89\end{array}$ \\
\hline $\begin{array}{l}\text { PROXIMATE } \\
\% \text { moisture } \\
\% \text { ash (dry) } \\
\text { analysis date } \\
\end{array}$ & $\begin{array}{c}9.6 \\
7.1 \\
03 / 13 / 86 \\
\end{array}$ & $\begin{array}{r}9.6 \\
7.3 \\
06 / 1.4 / 88 \\
\end{array}$ & $\begin{array}{c}9.8 \\
6.6 \\
02 / 01 / 89\end{array}$ & $\begin{array}{c}11.1 \\
7.1 \\
02 / 06 / 90\end{array}$ \\
\hline $\begin{array}{l}\text { SULFUR FORMS (dry \%) } \\
\text { total sulfur } \\
\text { sulfate } \\
\text { pyritic } \\
\text { organic } \\
\text { analysis date } \\
\end{array}$ & $\begin{array}{c}0.52 \\
0.00 \\
0.03 \\
0.48 \\
03 / 13 / 86 \\
\end{array}$ & & $\begin{array}{c}0.55 \\
0.02 \\
0.04 \\
0.49 \\
02 / 12 / 90 \\
\end{array}$ & $\begin{array}{c}0.46 \\
0.01 \\
0.02 \\
0.43 \\
02 / 06 / 90\end{array}$ \\
\hline $\begin{array}{l}\text { CALORIFIC VALUE (dry) } \\
\text { BTU/lb } \\
\text { analysis date }\end{array}$ & $\begin{array}{c}12762 \\
03 / 13 / 86 \\
\end{array}$ & & & $\begin{array}{c}12520 \\
02 / 06 / 90 \\
\end{array}$ \\
\hline $\begin{array}{l}\text { ALKALI EXTRACTION } \\
\% \text { transmission } \\
\text { analysis date }\end{array}$ & & $\begin{array}{c}23.5 \\
06 / 16 / 88\end{array}$ & $\begin{array}{c}38.8 \\
12 / 14 / 88\end{array}$ & $\begin{array}{l}\text { discon- } \\
\text { tinued }\end{array}$ \\
\hline
\end{tabular}

notes: a) Sample dry (dusty), good condition. PSU MCL ash, moisture; $S$ forms (revised). 


\begin{tabular}{|c|c|c|c|c|}
\hline $\begin{array}{l}\text { PSOC-1499 } \\
\text { collection date: }\end{array}$ & $\begin{array}{l}\text { OLORADO B } \\
9 / 26 / 85\end{array}$ & hvBb, & Ro $\max =$ & \\
\hline $\begin{array}{r}\text { Split ID } \\
\text { size } \\
\text { \% head oxygen } \\
\text { note } \\
\text { date sealed } \\
\text { date oxygen }\end{array}$ & $\begin{array}{l}\text { analysis } \\
\text { sample } 1 \\
-60 \text { mesh }\end{array}$ & $\begin{array}{l}\# 2 \text { can } \\
15 \text { of } 31 \\
-2 \text { mesh } \\
12 / 17 / 85\end{array}$ & $\begin{array}{c}30 \mathrm{gal} \\
3 \text { of } 4 \\
-1 / 4^{\prime \prime} \\
17.1 \\
a \\
10 / 14 / 88\end{array}$ & $\begin{array}{l}30 \text { ga } 1 \\
3 \text { of } 4 \\
-1 / 4^{11} \\
18.3 \\
10 / 11 / 89\end{array}$ \\
\hline $\begin{array}{l}\text { PROXIMATE } \\
\% \text { moisture } \\
\% \text { ash (dry) } \\
\text { analysis date }\end{array}$ & $\begin{array}{c}3.4 \\
5.9 \\
03 / 13 / 86 \\
\end{array}$ & $\begin{array}{c}4.2 \\
4.4 \\
04 / 22 / 88 \\
\end{array}$ & $\begin{array}{c}3.7 \\
5.3 \\
02 / 03 / 89 \\
\end{array}$ & $\begin{array}{c}4.4 \\
5.7 \\
02 / 28 / 90 \\
\end{array}$ \\
\hline $\begin{array}{l}\text { GIESELER FLUIDITY } \\
\text { max. ddpm } \\
\text { fluid range } \\
\text { analysis date }\end{array}$ & $\begin{array}{c}4 \\
43 \\
12 / 17 / 85 \\
\end{array}$ & $\begin{array}{r}4 \\
44 \\
04 / 22 / 88 \\
\end{array}$ & $\begin{array}{c}2 \\
36 \\
12 / 05 / 88 \\
\end{array}$ & $\begin{array}{c}1 \\
18 \\
02 / 01 / 90 \\
\end{array}$ \\
\hline $\begin{array}{l}\text { SULFUR FORMS (dry \%) } \\
\text { total sulfur } \\
\text { sulfate } \\
\text { pyritic } \\
\text { organic } \\
\text { analysis date }\end{array}$ & $\begin{array}{c}0.67 \\
0.00 \\
0.02 \\
0.65 \\
03 / 13 / 86\end{array}$ & & $\begin{array}{c}0.74 \\
0.04 \\
0.04 \\
0.66 \\
02 / 12 / 90 \\
\end{array}$ & $\begin{array}{r}0.64 \\
0.01 \\
0.02 \\
0.61 \\
02 / 28 / 90 \\
\end{array}$ \\
\hline $\begin{array}{l}\text { CALORIFIC VALUE (dry) } \\
\text { BTU/Ib } \\
\text { analysis date }\end{array}$ & $\begin{array}{c}13780 \\
03 / 13 / 86 \\
\end{array}$ & & & $\begin{array}{c}13597 \\
02 / 28 / 90 \\
\end{array}$ \\
\hline $\begin{array}{l}\text { ALKALI EXTRACTION } \\
\% \text { transmission } \\
\text { analysis date }\end{array}$ & & $\begin{array}{c}98.7 \\
06 / 06 / 88\end{array}$ & & $\begin{array}{c}91.8 \\
02 / 01 / 90\end{array}$ \\
\hline
\end{tabular}

notes: a) Sample dry (dusty), good condition. PSU MCL ash, moisture; $S$ forms (revised). 


\begin{tabular}{|c|c|c|c|c|}
\hline $\begin{array}{l}\text { PSOC- } 1500 \\
\text { collection date: }\end{array}$ & $\begin{array}{l}\text { ASIN B } \\
9 / 26 / 85\end{array}$ & $m v b$, & Ro $\max =$ & \\
\hline $\begin{array}{r}\text { Split I0 } \\
\text { size } \\
\% \text { head oxygen } \\
\text { note } \\
\text { date sealed } \\
\text { date oxygen }\end{array}$ & $\begin{array}{l}\text { analysis } \\
\text { sample } 1 \\
-60 \text { mesh }\end{array}$ & $\begin{array}{l}\# 2 \text { can } \\
15 \text { of } 31 \\
-20 \text { mesh } \\
02 / 11 / 86\end{array}$ & $\begin{array}{c}30 \text { gal } \\
2 \text { of } 4 \\
-1 / 4^{11} \\
19.7 \\
a \\
10 / 19 / 88\end{array}$ & $\begin{array}{l}30 \mathrm{gal} \\
2 \text { of } 4 \\
-1 / 4^{\prime \prime} \\
19.4 \\
10 / 11 / 89\end{array}$ \\
\hline $\begin{array}{l}\text { PROXIMATE } \\
\% \text { moisture } \\
\% \text { ash (dry) } \\
\text { analysis date }\end{array}$ & $\begin{array}{c}4.4 \\
23.4 \\
03 / 13 / 86 \\
\end{array}$ & $\begin{array}{c}2.2 \\
21.8 \\
04 / 25 / 88\end{array}$ & $\begin{array}{c}1.6 \\
24.2 \\
02 / 03 / 89\end{array}$ & $\begin{array}{c}2.8 \\
22.8 \\
03 / 01 / 90\end{array}$ \\
\hline $\begin{array}{l}\text { GIESELER FLUIDITY } \\
\text { max. ddpm } \\
\text { fluid range } \\
\text { analysis date } \\
\end{array}$ & $\begin{array}{r}267 \\
83 \\
03 / 13 / 86 \\
\end{array}$ & $\begin{array}{r}284 \\
84 \\
04 / 25 / 88 \\
\end{array}$ & $\begin{array}{c}30 \\
70 \\
01 / 17 / 89\end{array}$ & $\begin{array}{c}23 \\
66 \\
02 / 02 / 90\end{array}$ \\
\hline $\begin{array}{l}\text { SULFUR FORMS (dry \%) } \\
\text { total sulfur } \\
\text { sulfate } \\
\text { pyritic } \\
\text { organic } \\
\text { analysis date } \\
\end{array}$ & $\begin{array}{c}0.76 \\
0.00 \\
0.32 \\
0.44 \\
03 / 13 / 86 \\
\end{array}$ & & $\begin{array}{c}0.84 \\
0.13 \\
0.17 \\
0.54 \\
02 / 12 / 90 \\
\end{array}$ & $\begin{array}{c}0.74 \\
0.08 \\
0.19 \\
0.47 \\
03 / 01 / 90 \\
\end{array}$ \\
\hline $\begin{array}{l}\text { CALORIFIC VALUE (dry) } \\
\text { BTU/lb } \\
\text { analysis date }\end{array}$ & $\begin{array}{c}11986 \\
03 / 13 / 86 \\
\end{array}$ & & & $\begin{array}{c}12005 \\
03 / 01 / 90 \\
\end{array}$ \\
\hline $\begin{array}{l}\text { ALKALI EXTRACTION } \\
\% \text { transmission } \\
\text { analysis date }\end{array}$ & & $\begin{array}{c}99.0 \\
06 / 06 / 88\end{array}$ & & $\begin{array}{c}99.1 \\
02 / 02 / 90\end{array}$ \\
\hline
\end{tabular}

notes: a) Sample moist, yood condition. PSU MCL ash, moisture; $S$ forms (revised). 


\begin{tabular}{|c|c|c|c|c|}
\hline $\begin{array}{l}\text { PSOC-1501 } \\
\text { collection date: }\end{array}$ & $\begin{array}{l}\text { UANITA C } \\
9 / 27 / 85\end{array}$ & $h \cup B b$, & Ro $\max =$ & \\
\hline $\begin{array}{r}\text { Split ID } \\
\text { size } \\
\text { \% head oxygen } \\
\text { note } \\
\text { date sealed } \\
\text { date oxygen }\end{array}$ & $\begin{array}{l}\text { analysis } \\
\text { sample } 1 \\
-60 \text { mesh }\end{array}$ & $\begin{array}{l}\# 2 \text { can } \\
16 \text { of } 29 \\
-20 \text { mesh } \\
03 / 03 / 86\end{array}$ & $\begin{array}{c}30 \text { gal } \\
3 \text { of } 3 \\
-1 / 4^{11} \\
16.4 \\
10 / 19 / 88\end{array}$ & $\begin{array}{l}30 \text { gal } \\
3 \text { of } 3 \\
-1 / 4^{11} \\
17.7 \\
10 / 12 / 89\end{array}$ \\
\hline $\begin{array}{l}\text { PROXIMATE } \\
\% \text { moisture } \\
\% \text { ash (dry) } \\
\text { analysis date }\end{array}$ & $\begin{array}{c}5.8 \\
5.6 \\
03 / 13 / 86 \\
\end{array}$ & $\begin{array}{c}6.8 \\
5.4 \\
04 / 26 / 88 \\
\end{array}$ & $\begin{array}{c}6.0 \\
5.5 \\
02 / 10 / 89\end{array}$ & $\begin{array}{c}6.5 \\
5.6 \\
02 / 06 / 90\end{array}$ \\
\hline $\begin{array}{l}\text { GIESELER FLUIDITY } \\
\text { max. ddpm } \\
\text { fluid range } \\
\text { analysis date }\end{array}$ & $\begin{array}{r}1 \\
24 \\
03 / 20 / 86 \\
\end{array}$ & $\begin{array}{r}1 \\
25 \\
04 / 26 / 88 \\
\end{array}$ & $\begin{array}{c}0 \\
0 \\
02 / 02 / 89\end{array}$ & $\begin{array}{l}\text { discon- } \\
\text { tinued }\end{array}$ \\
\hline $\begin{array}{l}\text { SULFUR FORMS (dry \%) } \\
\text { total sulfur } \\
\text { sulfate } \\
\text { pyritic } \\
\text { organic } \\
\text { analysis date }\end{array}$ & $\begin{array}{c}0.64 \\
0.00 \\
0.02 \\
0.62 \\
03 / 13 / 86\end{array}$ & & $\begin{array}{c}0.57 \\
0.02 \\
0.03 \\
0.53 \\
02 / 10 / 89\end{array}$ & $\begin{array}{c}0.61 \\
0.01 \\
0.03 \\
0.57 \\
02 / 06 / 90\end{array}$ \\
\hline $\begin{array}{l}\text { CALORIFIC VALUE (dry) } \\
\text { BTU/lb } \\
\text { analysts date }\end{array}$ & $\begin{array}{c}13579 \\
03 / 13 / 86 \\
\end{array}$ & & $\begin{array}{c}13368 \\
02 / 10 / 89 \\
\end{array}$ & $\begin{array}{l}13342 \\
02 / 06 / 90\end{array}$ \\
\hline $\begin{array}{l}\text { ALKALI EXTRACTION } \\
\% \text { transmission } \\
\text { analysis date }\end{array}$ & & $\begin{array}{c}94.3 \\
06 / 06 / 88\end{array}$ & $\begin{array}{c}90.9 \\
02 / 10 / 89\end{array}$ & $\begin{array}{c}92.0 \\
01 / 23 / 90\end{array}$ \\
\hline
\end{tabular}




\begin{tabular}{|c|c|c|c|c|}
\hline $\begin{array}{l}\text { PSOC }-1502 \\
\quad \text { collection date: }\end{array}$ & $\begin{array}{l}\text { IAWATHA } \\
9 / 30 / 85\end{array}$ & hvCb, & Ro $\max =0$ & \\
\hline $\begin{array}{r}\text { Split ID } \\
\text { size } \\
\% \text { head oxygen } \\
\text { note } \\
\text { date sealed } \\
\text { date oxygen }\end{array}$ & $\begin{array}{l}\text { analysis } \\
\text { sample } 1 \\
-60 \text { mesh }\end{array}$ & $\begin{array}{l}\# 2 \text { Can } \\
14 \text { of } 29 \\
-20 \text { mesh } \\
03 / 03 / 86\end{array}$ & $\begin{array}{c}30 \text { gal } \\
2 \text { of } 4 \\
-111 \\
6.2 \\
10 / 19 / 88\end{array}$ & $\begin{array}{l}30 \text { gal. } \\
2 \text { of } 4 \\
-1 / 4^{11} \\
9.6 \\
10 / 12 / 89\end{array}$ \\
\hline $\begin{array}{l}\text { PROXIMATE } \\
\% \text { moisture } \\
\% \text { a sh (dry) } \\
\text { analysis date } \\
\end{array}$ & $\begin{array}{c}8.7 \\
9.0 \\
03 / 13 / 86 \\
\end{array}$ & $\begin{array}{c}8.4 \\
8.9 \\
06 / 14 / 88 \\
\end{array}$ & $\begin{array}{c}8.4 \\
9.0 \\
02 / 10 / 89\end{array}$ & $\begin{array}{c}8.9 \\
9.1 \\
02 / 06 / 90\end{array}$ \\
\hline $\begin{array}{l}\text { GIESELER FLUIDITY } \\
\text { max. ddpm } \\
\text { fluid range } \\
\text { analysis date }\end{array}$ & $\begin{array}{r}0 \\
0 \\
03 / 21 / 86\end{array}$ & $\begin{array}{l}\text { discon- } \\
\text { tinued }\end{array}$ & & \\
\hline $\begin{array}{l}\text { SULFUR FORMS (dry \%) } \\
\text { total sulfur } \\
\text { sulfate } \\
\text { pyritic } \\
\text { organic } \\
\text { analysis date } \\
\end{array}$ & $\begin{array}{r}0.51 \\
0.00 \\
0.15 \\
0.35 \\
03 / 13 / 86 \\
\end{array}$ & & $\begin{array}{c}0.43 \\
0.02 \\
0.13 \\
0.28 \\
02 / 10 / 89\end{array}$ & $\begin{array}{c}0.42 \\
0.01 \\
0.11 \\
0.29 \\
02 / 06 / 90\end{array}$ \\
\hline $\begin{array}{l}\text { CALORIFIC VALUE (dry) } \\
\text { BTU/lb } \\
\text { analysis date }\end{array}$ & $\begin{array}{c}12650 \\
03 / 13 / 86\end{array}$ & & $\begin{array}{c}12576 \\
02 / 10 / 89\end{array}$ & $\begin{array}{c}12574 \\
02 / 06 / 90\end{array}$ \\
\hline $\begin{array}{l}\text { ALKALI EXTRACTION } \\
\% \text { transmission } \\
\text { analysis date }\end{array}$ & & $\begin{array}{c}54.6 \\
06 / 15 / 88\end{array}$ & $\begin{array}{c}80.6 \\
11 / 10 / 89\end{array}$ & $\begin{array}{c}58.8 \\
01 / 23 / 90\end{array}$ \\
\hline
\end{tabular}




\begin{tabular}{|c|c|c|c|c|}
\hline $\begin{array}{l}\text { PSOC }-1503 \\
\text { collection date: }\end{array}$ & \multicolumn{4}{|c|}{$\begin{array}{l}\text { BLIND CANYON hvCb, Ro } \max =0.62 \\
10 / 01 / 85\end{array}$} \\
\hline $\begin{array}{r}\text { Split ID } \\
\text { size } \\
\% \text { head oxygen } \\
\text { mote } \\
\text { date sealed } \\
\text { date oxygen }\end{array}$ & $\begin{array}{l}\text { analysis } \\
\text { sample } 1 \\
-60 \text { mesh }\end{array}$ & $\begin{array}{l}\# 2 \text { can } \\
15 \text { of } 31 \\
-20 \text { mesh } \\
03 / 20 / 86\end{array}$ & $\begin{array}{c}30 \text { gal. } \\
3 \text { of } 4 \\
-111 \\
16.9 \\
10 / 19 / 88\end{array}$ & $\begin{array}{l}30 \text { gal. } \\
3 \text { of } 4 \\
-1 / 4^{\prime \prime} \\
16.6 \\
10 / 12 / 89\end{array}$ \\
\hline $\begin{array}{l}\text { PROXIMATE } \\
\% \text { moisture } \\
\% \text { ash (dry) } \\
\text { analysis date }\end{array}$ & $\begin{array}{c}10.4 \\
3.9 \\
04 / 25 / 86\end{array}$ & $\begin{array}{c}5.1 \\
4.0 \\
04 / 26 / 88\end{array}$ & $\begin{array}{c}5.2 \\
4.0 \\
02 / 10 / 89\end{array}$ & $\begin{array}{c}5.6 \\
4.1 \\
02 / 06 / 90\end{array}$ \\
\hline $\begin{array}{l}\text { GIESELER FLUIDITY } \\
\text { max. ddpm } \\
\text { fluid range } \\
\text { analysis date }\end{array}$ & $\begin{array}{c}2 \\
39 \\
03 / 24 / 86\end{array}$ & $\begin{array}{r}0 \\
0 \\
04 / 26 / 88\end{array}$ & $\begin{array}{l}\text { discon- } \\
\text { tinued }\end{array}$ & \\
\hline $\begin{array}{l}\text { SULFUR FORMS (dry \%) } \\
\text { total sulfur } \\
\text { sulfate } \\
\text { pyritic } \\
\text { organic } \\
\text { analysis date } \\
\end{array}$ & $\begin{array}{c}0.54 \\
0.00 \\
0.02 \\
0.52 \\
04 / 25 / 86 \\
\end{array}$ & & $\begin{array}{r}0.47 \\
0.01 \\
0.17 \\
0.29 \\
02 / 10 / 89 \\
\end{array}$ & $\begin{array}{r}0.48 \\
0.02 \\
0.02 \\
0.44 \\
02 / 06 / 90 \\
\end{array}$ \\
\hline $\begin{array}{l}\text { CALORIFIC VALUE (dry) } \\
\text { BTU/Ib } \\
\text { analysis date }\end{array}$ & $\begin{array}{c}14069 \\
04 / 25 / 86\end{array}$ & & $\begin{array}{c}13984 \\
02 / 10 / 89\end{array}$ & $\begin{array}{l}13832 \\
02 / 06 / 90\end{array}$ \\
\hline $\begin{array}{l}\text { ALKALI EXTRACTION } \\
\% \text { transmission } \\
\text { analysis date }\end{array}$ & & $\begin{array}{c}94.5 \\
06 / 06 / 88\end{array}$ & $\begin{array}{c}86.1 \\
02 / 10 / 89\end{array}$ & $\begin{array}{c}84.6 \\
01 / 24 / 90\end{array}$ \\
\hline
\end{tabular}




\begin{tabular}{|c|c|c|c|c|}
\hline \multirow{2}{*}{\begin{tabular}{|c} 
PSOC-1504 \\
collection date: \\
Split ID \\
size \\
$\%$ head oxygen \\
note \\
date sealed \\
date oxygen
\end{tabular}} & \multicolumn{4}{|c|}{$\begin{array}{l}\text { JPPER SUNNYSIDE hVBb, Ro } \max =0.80 \\
10 / 02.85\end{array}$} \\
\hline & $\begin{array}{l}\text { analysis } \\
\text { sample } 1 \\
-60 \text { mesh }\end{array}$ & $\begin{array}{l}\# 2 \text { can } \\
15 \text { of } 31 \\
-20 \text { mesh } \\
03 / 21 / 86\end{array}$ & $\begin{array}{c}30 \text { gal. } \\
4 \text { of } 4 \\
-1 / 4^{\prime \prime} \\
18.7 \\
a \\
03 / 18 / 86 \\
10 / 28 / 88\end{array}$ & $\begin{array}{l}30 \text { gal. } \\
4 \text { of } 4 \\
-1 / 4^{\prime \prime} \\
19.2 \\
10 / 12 / 89\end{array}$ \\
\hline $\begin{array}{l}\text { PROXIMATE } \\
\% \text { moisture } \\
\% \text { ash (dry) } \\
\text { analysis date }\end{array}$ & $\begin{array}{c}3.4 \\
7.5 \\
04 / 25 / 86\end{array}$ & $\begin{array}{c}2.8 \\
7.9 \\
05 / 04 / 88\end{array}$ & $\begin{array}{c}2.6 \\
7.3 \\
02 / 10 / 89\end{array}$ & $\begin{array}{c}3.0 \\
7.7 \\
02 / 06 / 90\end{array}$ \\
\hline $\begin{array}{l}\text { GIESELER FLUIDITY } \\
\text { max. ddpm } \\
\text { fluid range } \\
\text { analys is date }\end{array}$ & $\begin{array}{r}17 \\
51 \\
03 / 24 / 86\end{array}$ & $\begin{array}{r}13 \\
58 \\
05 / 04 / 88\end{array}$ & $\begin{array}{c}0 \\
0 \\
02 / 02 / 89\end{array}$ & $\begin{array}{l}\text { discon- } \\
\text { tinued }\end{array}$ \\
\hline $\begin{array}{l}\text { SULFUR FORMS (dry \%) } \\
\text { total sulfur } \\
\text { sulfate } \\
\text { pyritic } \\
\text { organic } \\
\text { analysis date }\end{array}$ & $\begin{array}{c}0.77 \\
0.02 \\
0.04 \\
0.71 \\
04 / 2.4 / 86\end{array}$ & & $\begin{array}{c}0.82 \\
0.02 \\
0.03 \\
0.77 \\
02 / 12 / 90\end{array}$ & $\begin{array}{c}0.73 \\
0.02 \\
0.02 \\
0.70 \\
02 / 06 / 90\end{array}$ \\
\hline $\begin{array}{l}\text { CALORIFIC VALUE (dry) } \\
\text { BTU/lb } \\
\text { analysis date }\end{array}$ & $\begin{array}{c}13640 \\
04 / 24 / 86\end{array}$ & & $\begin{array}{c}13612 \\
02 / 10 / 89\end{array}$ & $\begin{array}{c}13218 \\
02 / 06 / 90\end{array}$ \\
\hline $\begin{array}{l}\text { ALKALI EXTRACTION } \\
\% \text { transmission } \\
\text { analysis date }\end{array}$ & & $\begin{array}{c}97.2 \\
06 / 07 / 88\end{array}$ & $\begin{array}{c}91.8 \\
02 / 10 / 89\end{array}$ & $\begin{array}{r}87.4 \\
01 / 24 / 90\end{array}$ \\
\hline
\end{tabular}

notes: a) Sample dry, good condition. PSU S forms (revised). 


\begin{tabular}{|c|c|c|c|c|}
\hline $\begin{array}{l}\text { PSOC- } 1506 \\
\text { collection date: }\end{array}$ & $\begin{array}{l}\text { BANNER } \\
06 / 14 / 85\end{array}$ & hvAb, & Ro $\max =$ & \\
\hline $\begin{array}{r}\text { Split ID } \\
\text { size } \\
\% \text { head oxygen } \\
\text { note } \\
\text { date sealed } \\
\text { date oxygen }\end{array}$ & $\begin{array}{l}\text { analysis } \\
\text { sample } 1 \\
-60 m e s h\end{array}$ & $\begin{array}{l}\# 2 \text { can } \\
15 \text { of } 31 \\
-20 \text { mesh } \\
04 / 24 / 86\end{array}$ & $\begin{array}{c}30 \text { gal. } \\
3 \text { of } 3 \\
-1 / 4 " \\
19.4 \\
a \\
04 / 22 / 86 \\
10 / 28 / 88\end{array}$ & $\begin{array}{l}30 \text { gal. } \\
3 \text { of } 3 \\
-1 / 4 " \\
19.8 \\
10 / 13 / 89\end{array}$ \\
\hline $\begin{array}{l}\text { PROXIMATE } \\
\% \text { moisture } \\
\% \text { ash (dry) } \\
\text { analysis date }\end{array}$ & $\begin{array}{c}1.3 \\
13.6 \\
10 / 04 / 85\end{array}$ & $\begin{array}{c}1.8 \\
14.0 \\
05 / 05 / 88\end{array}$ & $\begin{array}{c}1.1 \\
14.7 \\
02 / 10 / 89\end{array}$ & $\begin{array}{c}1.5 \\
14.5 \\
03 / 01 / 90\end{array}$ \\
\hline $\begin{array}{l}\text { GIESELER FLUIDITY } \\
\text { max. ddpm } \\
\text { fluid range } \\
\text { analysis date }\end{array}$ & $\begin{array}{r}29881 \\
90 \\
04 / 25 / 86\end{array}$ & $\begin{array}{r}6645 \\
89 \\
05 / 05 / 88\end{array}$ & $\begin{array}{c}4889 \\
79 \\
02 / 02 / 89\end{array}$ & $\begin{array}{c}2870 \\
81 \\
02 / 09 / 90\end{array}$ \\
\hline $\begin{array}{l}\text { SULFUR FORMS (dry \%) } \\
\text { total sulfur } \\
\text { sulfate } \\
\text { pyritic } \\
\text { organic } \\
\text { analysis date } \\
\end{array}$ & $\begin{array}{c}0.70 \\
0.02 \\
0.14 \\
0.54 \\
10 / 0.5 / 85 \\
\end{array}$ & & $\begin{array}{c}0.72 \\
0.05 \\
0.07 \\
0.60 \\
02 / 12 / 90\end{array}$ & $\begin{array}{c}0.65 \\
0.06 \\
0.08 \\
0.51 \\
03 / 01 / 90\end{array}$ \\
\hline $\begin{array}{l}\text { CALORIFIC VALUE } \\
\text { BTU/ID dry } \\
\text { analysis date }\end{array}$ & $\begin{array}{c}13325 \\
10 / 05 / 85 \\
\end{array}$ & & $\begin{array}{c}13024 \\
02 / 10 / 89\end{array}$ & $\begin{array}{c}13095 \\
03 / 01 / 90\end{array}$ \\
\hline $\begin{array}{c}\text { ALKALI EXTRACTION } \\
\% \text { transmission } \\
\text { analysis date }\end{array}$ & & $\begin{array}{c}98.8 \\
06 / 08 / 88\end{array}$ & $\begin{array}{c}95.4 \\
02 / 13 / 89\end{array}$ & $\begin{array}{c}98.9 \\
02 / 09 / 90\end{array}$ \\
\hline
\end{tabular}

notes: a) Sample dry, good condition; PSU S forms (revised). 


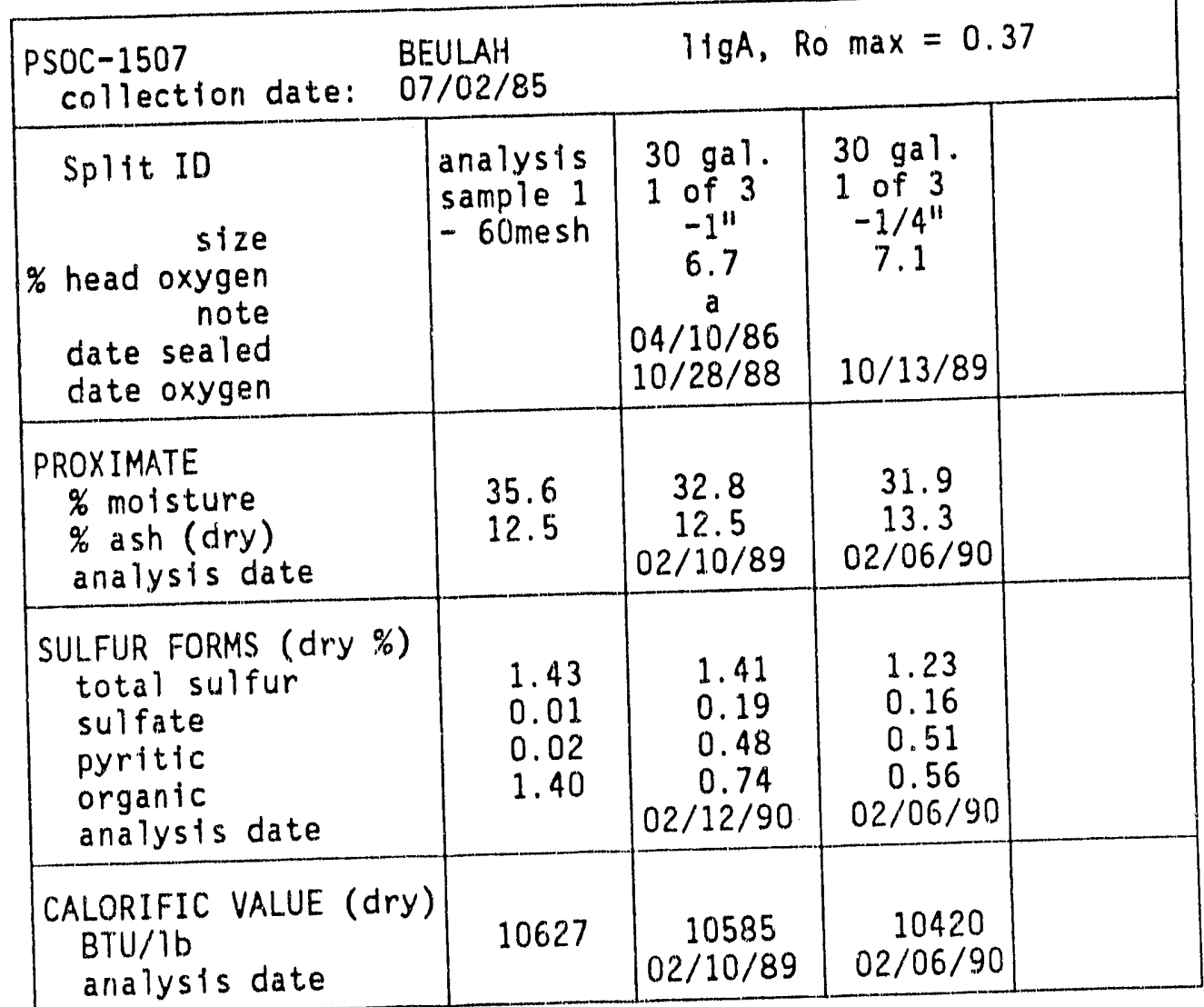

notes: a) Sample moist, trace white and yellow sulfate. PSU S forms (revised). 


\begin{tabular}{|c|c|c|c|c|}
\hline $\begin{array}{l}\text { PSOC-1508 } \\
\text { collection date: }\end{array}$ & $\begin{array}{l}\text { OCAHONTAS } \\
1 / 05 / 85\end{array}$ & \#3 Ivb, & Ro $\max =1$ & \\
\hline $\begin{array}{r}\text { Split ID } \\
\text { size } \\
\% \text { head oxygen } \\
\text { note } \\
\text { date sealed } \\
\text { date oxygen } \\
\end{array}$ & $\begin{array}{l}\text { analysis } \\
\text { sample } 1 \\
-60 \text { mesh }\end{array}$ & $\begin{array}{l}\# 2 \text { can } \\
15 \text { of } 31 \\
-20 \text { mesh } \\
12 / 04 / 85\end{array}$ & $\begin{array}{c}30 \mathrm{gal} \\
6 \text { of } 6 \\
-1 / 4^{\prime \prime} \\
20.7 \\
a \\
11 / 14 / 85 \\
10 / 28 / 88 \\
\end{array}$ & $\begin{array}{l}30 \text { ga } 1 \\
6 \text { of } 6 \\
-1 / 4^{\prime \prime} \\
20.9 \\
\\
10 / 13 / 89\end{array}$ \\
\hline $\begin{array}{l}\text { PROXIMATE } \\
\% \text { moisture } \\
\% \text { ash (dry) } \\
\text { analysis date }\end{array}$ & $\begin{array}{c}1.1 \\
7.4 \\
12 / 23 / 85 \\
\end{array}$ & $\begin{array}{c}1.9 \\
7.1 \\
05 / 06 / 88\end{array}$ & $\begin{array}{c}1.4 \\
7.4 \\
02 / 10 / 89\end{array}$ & $\begin{array}{c}1.3 \\
7.5 \\
02 / 06 / 90\end{array}$ \\
\hline $\begin{array}{l}\text { GIESELER FLUIDITY } \\
\text { max. ddpm } \\
\text { fluid range } \\
\text { analysis date }\end{array}$ & $\begin{array}{c}10 \\
44 \\
12 / 07 / 85\end{array}$ & $\begin{array}{r}3 \\
41 \\
05 / 06 / 88\end{array}$ & $\begin{array}{c}0 \\
0 \\
02 / 02 / 89\end{array}$ & $\begin{array}{l}\text { discon- } \\
\text { tinued }\end{array}$ \\
\hline $\begin{array}{l}\text { SULFUR FORMS (dry } \%) \\
\text { total sulfur } \\
\text { sulfate } \\
\text { pyritic } \\
\text { organic } \\
\text { analysis date }\end{array}$ & $\begin{array}{c}0.64 \\
0.00 \\
0.14 \\
0.50 \\
12 / 23 / 85 \\
\end{array}$ & & $\begin{array}{c}0.57 \\
0.02 \\
0.24 \\
0.31 \\
02 / 10 / 89\end{array}$ & $\begin{array}{c}0.61 \\
0.03 \\
0.07 \\
0.51 \\
02 / 06 / 90\end{array}$ \\
\hline $\begin{array}{l}\text { CALORIFIC VALUE (dry) } \\
\text { BTU/Ib } \\
\text { analysis date }\end{array}$ & $\begin{array}{c}14542 \\
12 / 23 / 85\end{array}$ & & $\begin{array}{c}14489 \\
02 / 10 / 89\end{array}$ & $\begin{array}{c}14345 \\
02 / 06 / 90\end{array}$ \\
\hline $\begin{array}{c}\text { ALKALI EXTRACTION } \\
\% \text { transmission } \\
\text { analysis date }\end{array}$ & & $\begin{array}{c}100.2 \\
06 / 09 / 88\end{array}$ & $\begin{array}{c}96.9 \\
02 / 13 / 89\end{array}$ & $\begin{array}{c}97.7 \\
01 / 25 / 90\end{array}$ \\
\hline
\end{tabular}

notes: a) Sample dry, good condition. 


\begin{tabular}{|c|c|c|c|c|}
\hline $\begin{array}{l}\text { PSOC- } 1515 \\
\text { collection date: }\end{array}$ & $\begin{array}{l}\text { ENNSYLVAN } \\
5 / 08 / 86\end{array}$ & & sa, Ro max & $=2.80$ \\
\hline $\begin{array}{r}\text { Split ID } \\
\text { size } \\
\% \text { head oxygen } \\
\text { note } \\
\text { date sealed } \\
\text { date oxygen }\end{array}$ & $\begin{array}{l}\text { analysis } \\
\text { sample } 1 \\
-60 \text { mesh }\end{array}$ & $\begin{array}{c}30 \text { gal. } \\
4 \text { of } 4 \\
-1 / 4^{\prime \prime} \\
19.8 \\
a \\
08 / 08 / 86 \\
10 / 28 / 88\end{array}$ & $\begin{array}{l}30 \text { gal. } \\
4 \text { of } 4 \\
-1 / 4^{\prime \prime} \\
20.3 \\
10 / 13 / 89\end{array}$ & \\
\hline $\begin{array}{l}\text { PROXIMATE } \\
\% \text { moisture } \\
\% \text { ash (dry) } \\
\text { analysis date }\end{array}$ & $\begin{array}{c}2.4 \\
29.2 \\
09 / 25 / 86 \\
\end{array}$ & $\begin{array}{c}2.2 \\
28.3 \\
02 / 10 / 89 \\
\end{array}$ & $\begin{array}{c}2.1 \\
28.3 \\
02 / 06 / 90 \\
\end{array}$ & \\
\hline $\begin{array}{l}\text { SULFUR FORMS (dry \%) } \\
\text { total sulfur } \\
\text { sulfate } \\
\text { pyritic } \\
\text { organic } \\
\text { analysis date }\end{array}$ & $\begin{array}{c}0.58 \\
0.00 \\
0.10 \\
0.49 \\
09 / 25 / 86\end{array}$ & $\begin{array}{c}0.63 \\
0.13 \\
0.04 \\
0.46 \\
02 / 12 / 90\end{array}$ & $\begin{array}{c}0.59 \\
0.10 \\
0.06 \\
0.43 \\
02 / 06 / 90\end{array}$ & \\
\hline $\begin{array}{l}\text { CALORIFIC VALUE (dry) } \\
\text { BTU/Ib } \\
\text { analysis date }\end{array}$ & $\begin{array}{c}10642 \\
09 / 16 / 86\end{array}$ & $\begin{array}{c}10677 \\
02 / 10 / 89\end{array}$ & $\begin{array}{c}10657 \\
02 / 06 / 90\end{array}$ & \\
\hline
\end{tabular}

notes: a) Sample moist, good condition. PSU S forms (revised). 


\begin{tabular}{|c|c|c|c|c|}
\hline \multicolumn{5}{|c|}{$\begin{array}{l}\text { PSOC- } 1516 \quad \text { LOWER KITTANNING IVb, } \text { Ro max }=1.73 \\
\text { collection date: } 05 / 29 / 86\end{array}$} \\
\hline $\begin{array}{r}\text { Split ID } \\
\text { size } \\
\% \text { head oxygen } \\
\text { note } \\
\text { date sealed } \\
\text { date oxygen } \\
\end{array}$ & $\begin{array}{l}\text { analysis } \\
\text { sample } 1 \\
-60 \text { mesh }\end{array}$ & $\begin{array}{l}\# 2 \text { can } \\
15 \text { of } 31 \\
-20 \text { mesh } \\
08 / 20 / 86\end{array}$ & $\begin{array}{c}30 \text { gal } \\
4 \text { of } 4 \\
-1 / 4^{\prime \prime} \\
20.5 \\
a \\
08 / 08 / 86 \\
10 / 28 / 88 \\
\end{array}$ & $\begin{array}{l}30 \text { gal. } \\
4 \text { of } 4 \\
-1 / 4^{\prime \prime} \\
20.6 \\
10 / 13 / 89 \\
\end{array}$ \\
\hline $\begin{array}{l}\text { PROXIMATE } \\
\% \text { moisture } \\
\% \text { ash (dry) } \\
\text { analysis date }\end{array}$ & $\begin{array}{c}2.0 \\
10.3 \\
09 / 25 / 86 \\
\end{array}$ & $\begin{array}{r}2.1 \\
9.4 \\
05 / 09 / 88 \\
\end{array}$ & $\begin{array}{c}2.1 \\
10.3 \\
02 / 10 / 89 \\
\end{array}$ & $\begin{array}{r}1.9 \\
10.5 \\
02 / 28 / 90 \\
\end{array}$ \\
\hline $\begin{array}{l}\text { GIESELER FLUIDITY } \\
\text { max. ddpm } \\
\text { fluid range } \\
\text { analysis date } \\
\end{array}$ & $\begin{array}{r}53 \\
69 \\
08 / 20 / 86 \\
\end{array}$ & $\begin{array}{r}2 \\
25 \\
05 / 09 / 88 \\
\end{array}$ & $\begin{array}{c}2 \\
\text { nd } \\
02 / 06 / 89\end{array}$ & $\begin{array}{l}0 \\
0\end{array}$ \\
\hline $\begin{array}{l}\text { SULFUR FORMS (dry \%) } \\
\text { total sulfur } \\
\text { sulfate } \\
\text { pyritic } \\
\text { organic } \\
\text { analysis date }\end{array}$ & $\begin{array}{c}1.40 \\
0.01 \\
0.61 \\
0.78 \\
09 / 25 / 86 \\
\end{array}$ & & $\begin{array}{r}1.33 \\
0.01 \\
0.81 \\
0.50 \\
02 / 10 / 89 \\
\end{array}$ & $\begin{array}{r}1.44 \\
0.29 \\
0.36 \\
0.79 \\
02 / 28 / 90 \\
\end{array}$ \\
\hline $\begin{array}{l}\text { CALORIFIC VALUE (dry) } \\
\text { BTU/lb } \\
\text { analysis date }\end{array}$ & $\begin{array}{r}14025 \\
09 / 25 / 86 \\
\end{array}$ & & $\begin{array}{c}13822 \\
02 / 10 / 89 \\
\end{array}$ & $\begin{array}{r}13752 \\
02 / 28 / 90 \\
\end{array}$ \\
\hline $\begin{array}{c}\text { ALKALI EXTRACTION } \\
\% \text { transmission } \\
\text { analysis date }\end{array}$ & & $\begin{array}{c}99.9 \\
06 / 08 / 88\end{array}$ & $\begin{array}{c}95.9 \\
02 / 15 / 89 \\
\end{array}$ & $\begin{array}{c}98.8 \\
02 / 12 / 90 \\
\end{array}$ \\
\hline
\end{tabular}

notes: a) Sample dry, good condition. 


\begin{tabular}{|c|c|c|c|c|}
\hline $\begin{array}{l}\text { PSOC-1517 } \\
\text { collection date: }\end{array}$ & $\begin{array}{l}0 \# 5 \\
09 / 86\end{array}$ & huBb, & Ro $\max =$ & \\
\hline $\begin{array}{r}\text { Split ID } \\
\text { size } \\
\% \text { head oxygen } \\
\text { note } \\
\text { date sealed } \\
\text { date oxygen }\end{array}$ & $\begin{array}{l}\text { analysis } \\
\text { sample } 1 \\
-60 \text { mesh }\end{array}$ & $\begin{array}{l}\# 2 \text { can } \\
16 \text { of } 31 \\
-20 \text { mesh } \\
08 / 2 ? / 86\end{array}$ & $\begin{array}{c}30 \text { gal } \\
2 \text { of } 3 \\
-111 \\
16.4 \\
08 / 07 / 86 \\
10 / 28 / 88\end{array}$ & $\begin{array}{l}30 \text { ga } 1 \\
2 \text { of } 3 \\
-1 / 4^{11} \\
18.7 \\
10 / 13 / 89\end{array}$ \\
\hline $\begin{array}{l}\text { PROXIMATE } \\
\% \text { moisture } \\
\% \text { ash (dry) } \\
\text { analysis date }\end{array}$ & $\begin{array}{c}4.2 \\
4.8 \\
09 / 25 / 86\end{array}$ & $\begin{array}{c}4.0 \\
4.7 \\
05 / 10 / 88\end{array}$ & $\begin{array}{c}4.3 \\
5.8 \\
02 / 10 / 89\end{array}$ & $\begin{array}{c}4.5 \\
5.7 \\
02 / 28 / 90\end{array}$ \\
\hline $\begin{array}{l}\text { GIESELER FLUIDITY } \\
\text { max. ddpm } \\
\text { fluid range } \\
\text { analysis date } \\
\end{array}$ & $\begin{array}{c}1509 \\
75 \\
09 / 03 / 86\end{array}$ & $\begin{array}{c}13 \\
50 \\
05 / 10 / 88\end{array}$ & $\begin{array}{c}77 \\
60 \\
02 / 06 / 89\end{array}$ & $\begin{array}{c}18 \\
52 \\
02 / 13 / 90\end{array}$ \\
\hline $\begin{array}{l}\text { SULFUR FORMS (dry \%) } \\
\text { total sulfur } \\
\text { sulfate } \\
\text { pyritic } \\
\text { organic } \\
\text { analysis date } \\
\end{array}$ & $\begin{array}{c}1.92 \\
0.01 \\
0.70 \\
1.21 \\
09 / 25 / 86 \\
\end{array}$ & & $\begin{array}{r}2.09 \\
0.01 \\
0.98 \\
1.10 \\
02 / 10 / 89 \\
\end{array}$ & $\begin{array}{r}2.19 \\
0.32 \\
1.17 \\
0.70 \\
02 / 28 / 90 \\
\end{array}$ \\
\hline $\begin{array}{l}\text { CALORIFIC VALUE (dry) } \\
\text { BTU/lb } \\
\text { analysis date }\end{array}$ & $\begin{array}{c}14258 \\
09 / 25 / 86\end{array}$ & & $\begin{array}{c}13933 \\
02 / 10 / 89\end{array}$ & $\begin{array}{c}13841 \\
02 / 28 / 90\end{array}$ \\
\hline $\begin{array}{l}\text { ALKALI EXTRACTION } \\
\% \text { transmisston } \\
\text { analysis date }\end{array}$ & & $\begin{array}{c}98.0 \\
06 / 09 / 88\end{array}$ & $\begin{array}{c}98.1 \\
02 / 15 / 89\end{array}$ & $\begin{array}{c}87.0 \\
02 / 13 / 90\end{array}$ \\
\hline
\end{tabular}

notes: a) Sample dry, good condition. 


\begin{tabular}{|c|c|c|c|c|}
\hline \multicolumn{5}{|c|}{$\begin{array}{l}\text { PSOC- } 1: 18 \quad \text { M. KITTANNING (OHIO \#6) hvBb, Ro max }=0.69 \\
\text { collection date: } 06 / 10 / 86\end{array}$} \\
\hline $\begin{array}{r}\text { Split ID } \\
\text { size } \\
\text { \% head oxygen } \\
\text { note } \\
\text { date sealed } \\
\text { date oxygen }\end{array}$ & $\begin{array}{l}\text { analysis } \\
\text { sample } 1 \\
-60 \text { mesh }\end{array}$ & $\begin{array}{l}\# 2 \text { can } \\
15 \text { of } 31 \\
-20 \text { mesh } \\
08 / 26 / 86\end{array}$ & $\begin{array}{c}30 \text { gal } \\
3 \text { of } 3 \\
-1 / 4^{\prime \prime} \\
18.5 \\
\text { a } \\
08 / 07 / 86 \\
10 / 28 / 88\end{array}$ & $\begin{array}{c}30 \text { gal } \\
3 \text { of } 3 \\
-1 / 4^{11} \\
19.1 \\
10 / 16 / 89\end{array}$ \\
\hline $\begin{array}{l}\text { PROXIMATE } \\
\% \text { moisture } \\
\% \text { ash (dry) } \\
\text { analysis date }\end{array}$ & $\begin{array}{c}5.3 \\
9.9 \\
09 / 25 / 86 \\
\end{array}$ & $\begin{array}{c}5.0 \\
9.3 \\
05 / 11 / 88\end{array}$ & $\begin{array}{c}5.8 \\
9.6 \\
03 / 07 / 89\end{array}$ & $\begin{array}{c}5.9 \\
9.5 \\
02 / 06 / 90\end{array}$ \\
\hline $\begin{array}{l}\text { GIESELER FLUIDITY } \\
\text { max. ddpm } \\
\text { fluid range } \\
\text { analys is date } \\
\end{array}$ & $\begin{array}{r}113 \\
62 \\
09 / 04 / 86 \\
\end{array}$ & $\begin{array}{r}118 \\
66 \\
05 / 11 / 88\end{array}$ & & \\
\hline $\begin{array}{l}\text { SULFUR FORMS (dry \%) } \\
\text { total sulfur } \\
\text { sulfate } \\
\text { pyritic } \\
\text { organic } \\
\text { analysis date } \\
\end{array}$ & $\begin{array}{r}3.86 \\
0.12 \\
2.26 \\
1.48 \\
09 / 2 ! / 86 \\
\end{array}$ & & $\begin{array}{c}3.44 \\
0.91 \\
1.05 \\
1.48 \\
03 / 07 / 89 \\
\end{array}$ & $\begin{array}{r}3.77 \\
1.06 \\
0.88 \\
1.83 \\
02 / 06 / 90 \\
\end{array}$ \\
\hline $\begin{array}{l}\text { CALORIFIC VALUE (dry) } \\
\text { BTU/Ib } \\
\text { analysis date }\end{array}$ & $\begin{array}{c}13074 \\
09 / 25 / 86 \\
\end{array}$ & & $\begin{array}{r}12258 \\
03 / 07 / 89 \\
\end{array}$ & $\begin{array}{c}12538 \\
02 / 06 / 90 \\
\end{array}$ \\
\hline $\begin{array}{l}\text { ALKALI EXTRACTION } \\
\text { \% transmission } \\
\text { analysis date }\end{array}$ & & $\begin{array}{c}97.4 \\
06 / 09 / 88\end{array}$ & $\begin{array}{c}94.8 \\
03 / 06 / 89\end{array}$ & $\begin{array}{c}92.4 \\
01 / 25 / 90\end{array}$ \\
\hline
\end{tabular}

notes: a) Sample dry, large visible white sulfate crystais. 


\begin{tabular}{|c|c|c|c|c|}
\hline $\begin{array}{l}\text { PSOC- } 1519 \\
\text { collection date: }\end{array}$ & $\begin{array}{l}\text { ITTSBURGH } \\
6 / 19 / 86\end{array}$ & hvBb, & Ro $\max =$ & \\
\hline $\begin{array}{r}\text { Split ID } \\
\text { slze } \\
\% \text { head oxygen } \\
\text { note } \\
\text { date sealed } \\
\text { date oxygen }\end{array}$ & $\begin{array}{l}\text { analysis } \\
\text { sample } 1 \\
-60 \text { mesh }\end{array}$ & $\begin{array}{l}\# 2 \text { can } \\
15 \text { of } 31 \\
-20 \text { mesh } \\
08 / 29 / 86\end{array}$ & $\begin{array}{c}30 \text { gal. } \\
1 \text { of } 3 \\
-1 / 4^{\prime \prime} \\
18.3 \\
\text { a } \\
8 / 06 / 86 \\
9 / 24 / 88\end{array}$ & $\begin{array}{l}30 \mathrm{gal} \\
1 \text { of } 3 \\
-1 / 4^{11} \\
18.4 \\
10 / 16 / 89\end{array}$ \\
\hline $\begin{array}{l}\text { PROXIMATic } \\
\% \text { moisture } \\
\% \text { ash (dry) } \\
\text { analysis date }\end{array}$ & $\begin{array}{c}3.1 \\
10.5 \\
09 / 25 / 86\end{array}$ & $\begin{array}{c}3.5 \\
9.8 \\
05 / 12 / 88\end{array}$ & $\begin{array}{c}1.5 \\
10.2 \\
10 / 12 / 88\end{array}$ & $\begin{array}{c}3.3 \\
11.7 \\
02 / 28 / 90\end{array}$ \\
\hline $\begin{array}{l}\text { GIESELER FLUIDITY } \\
\text { max. ddpm } \\
\text { fluid range } \\
\text { analysis date } \\
\end{array}$ & $\begin{array}{c}3548 \\
77 \\
12 / 15 / 86 \\
\end{array}$ & $\begin{array}{r}1409 \\
80 \\
05 / 12 / 88 \\
\end{array}$ & $\begin{array}{c}800 \\
67 \\
10 / 21 / 88 \\
\end{array}$ & $\begin{array}{r}17 \\
39 \\
02 / 14 / 90 \\
\end{array}$ \\
\hline $\begin{array}{l}\text { SULFUR FORMS (dry \%) } \\
\text { total sulfur } \\
\text { sulfate } \\
\text { pyritic } \\
\text { organic } \\
\text { analysis date } \\
\end{array}$ & $\begin{array}{c}1.94 \\
0.10 \\
0.02 \\
1.82 \\
09 / 25 / 86 \\
\end{array}$ & & $\begin{array}{c}1.93 \\
0.47 \\
0.60 \\
0.86 \\
09 / 17 / 89 \\
\end{array}$ & $\begin{array}{r}1.97 \\
0.55 \\
0.42 \\
1.00 \\
02 / 28 / 90 \\
\end{array}$ \\
\hline $\begin{array}{l}\text { CALORIFIC VALUE (dry) } \\
\text { BTU/lb } \\
\text { analysis date }\end{array}$ & $\begin{array}{c}13382 \\
09 / 25 / 86\end{array}$ & & & $\begin{array}{l}12959 \\
02 / 28 / 90\end{array}$ \\
\hline $\begin{array}{l}\text { ALKALI EXTRACTION } \\
\% \text { transmission } \\
\text { analysis date }\end{array}$ & & $\begin{array}{c}81.4 \\
06 / 09 / 88\end{array}$ & $\begin{array}{c}85.0 \\
10 / 06 / 88\end{array}$ & $\begin{array}{c}70.7 \\
02 / 28 / 90\end{array}$ \\
\hline
\end{tabular}

notes: a) PSU MCL ash, moisture. 


\begin{tabular}{|c|c|c|c|c|}
\hline $\begin{array}{l}\text { PSOC-1520 } \\
\text { collection date: }\end{array}$ & \multicolumn{4}{|c|}{$\begin{array}{l}\text { SMITH ROLAND } \quad \text { subC, Ro } \max =0.43 \\
08 / 18 / 86\end{array}$} \\
\hline $\begin{array}{r}\text { Split ID } \\
\text { size } \\
\% \text { head oxygen } \\
\text { note } \\
\text { date sealed } \\
\text { date oxygen }\end{array}$ & $\begin{array}{l}\text { analysis } \\
\text { sample } 1 \\
-60 \text { mesh }\end{array}$ & $\begin{array}{l}\text { analysis } \\
\text { sample } 2 \\
-60 \text { mesh }\end{array}$ & $\begin{array}{c}5 \mathrm{gal} \\
-1 / 4^{\prime \prime} \\
\text { a }\end{array}$ & $\begin{array}{l}30 \text { gal. } \\
4 \text { of } 5 \\
-111 \\
12.1 \\
10 / 16 / 89\end{array}$ \\
\hline $\begin{array}{l}\text { PROXIMATE } \\
\% \text { moisture } \\
\% \text { ash (dry) } \\
\text { analysis date }\end{array}$ & $\begin{array}{c}29.7 \\
12.4 \\
09 / 16 / 86\end{array}$ & $\begin{array}{c}26.7 \\
12.6 \\
10 / 05 / 87\end{array}$ & $\begin{array}{c}24.8 \\
10.5 \\
09 / 14 / 88\end{array}$ & $\begin{array}{c}27.3 \\
12.7 \\
02 / 06 / 90\end{array}$ \\
\hline $\begin{array}{l}\text { SULFUR FORMS (dry \%) } \\
\text { total sulfur } \\
\text { sulfate } \\
\text { pyritic } \\
\text { organic } \\
\text { analysis date }\end{array}$ & $\begin{array}{r}1.21 \\
0.01 \\
0.05 \\
1.15 \\
09 / 16 / 86 \\
\end{array}$ & $10 / 05 / 87$ & $\begin{array}{c}1.24 \\
0.34 \\
0.14 \\
0.76 \\
09 / 22 / 88\end{array}$ & $\begin{array}{c}1.18 \\
0.24 \\
0.23 \\
0.71 \\
02 / 06 / \mathrm{C} 0\end{array}$ \\
\hline $\begin{array}{l}\text { CALORIFIC VALUE (dry) } \\
\text { BTU/Ib } \\
\text { analysis date }\end{array}$ & $\begin{array}{c}11291 \\
09 / 16 / 86\end{array}$ & & & $\begin{array}{c}.1006 \\
02 / 06 / 90\end{array}$ \\
\hline
\end{tabular}

notes: a) Entire sample used for restocking \#2 cans. PSU MCL ash, moisture, sulfur forms. 


\begin{tabular}{|c|c|c|c|c|}
\hline $\begin{array}{l}\text { PSOC-1521 } \\
\text { collection date: }\end{array}$ & $\begin{array}{l}\text { HARTSHC } \\
8 / 22 / 86\end{array}$ & Ivb, & Ro $\max =1$ & \\
\hline $\begin{array}{r}\text { Split ID } \\
\text { size } \\
\% \text { head oxygen } \\
\text { note } \\
\text { date sealed } \\
\text { date oxygen }\end{array}$ & $\begin{array}{l}\text { analysis } \\
\text { sample } 1 \\
-60 \text { mesh }\end{array}$ & $\begin{array}{l}\# 2 \text { can } \\
15 \text { of } 31 \\
-2 \text { mesh } \\
08 / 29 / 86\end{array}$ & $\begin{array}{c}30 \text { ga } 1 \\
3 \text { of } 3 \\
-1 / 4^{\prime \prime} \\
18.8 \\
\text { a } \\
08 / 07 / 86 \\
10 / 28 / 88\end{array}$ & $\begin{array}{l}30 \text { gal. } \\
3 \text { of } 3 \\
-1 / 4^{11} \\
19.7 \\
10 / 23 / 89\end{array}$ \\
\hline $\begin{array}{l}\text { PROXIMATE } \\
\% \text { moisture } \\
\% \text { ash (dry) } \\
\text { anaiysis date }\end{array}$ & $\begin{array}{c}2.7 \\
11.2 \\
09 / 26 / 86\end{array}$ & $\begin{array}{c}2.1 \\
10.0 \\
05 / 13 / 88\end{array}$ & $\begin{array}{c}2.5 \\
11.4 \\
03 / 07 / 89\end{array}$ & $\begin{array}{c}2.6 \\
10.0 \\
02 / 06 / 90\end{array}$ \\
\hline $\begin{array}{l}\text { GIESELER FLUIDITY } \\
\text { max. ddpm } \\
\text { fluid range } \\
\text { analysis date }\end{array}$ & $\begin{array}{c}4 \\
38 \\
09 / 15 / 86\end{array}$ & $\begin{array}{c}0 \\
0 \\
05 / 13 / 38\end{array}$ & $\begin{array}{l}\text { discon- } \\
\text { tinued }\end{array}$ & \\
\hline $\begin{array}{l}\text { SULFUR FORMS (dry \%) } \\
\text { total sulfur } \\
\text { sulfate } \\
\text { pyritic } \\
\text { organic } \\
\text { analysis date } \\
\end{array}$ & $\begin{array}{c}0.80 \\
0.00 \\
0.02 \\
0.77 \\
09 / 26 / 86 \\
\end{array}$ & & $\begin{array}{c}0.79 \\
0.19 \\
0.16 \\
0.43 \\
03 / 07 / 89\end{array}$ & $\begin{array}{r}0.85 \\
0.18 \\
0.15 \\
0.52 \\
02 / 06 / 90\end{array}$ \\
\hline $\begin{array}{l}\text { CALORIFIC VALUE (dry) } \\
\text { BTU/Ib } \\
\text { analysis date }\end{array}$ & $\begin{array}{c}13612 \\
09 / 26 / 86\end{array}$ & & $\begin{array}{c}13538 \\
03 / 07 / 89 \\
\end{array}$ & $\begin{array}{r}13825 \\
02 / 06 / 90 \\
\end{array}$ \\
\hline $\begin{array}{l}\text { ALKALI EXTRACTION } \\
\% \text { transmission } \\
\text { analysis date }\end{array}$ & & $\begin{array}{c}99.5 \\
06 / 10 / 88\end{array}$ & $\begin{array}{c}96.1 \\
03 / 06 / 89\end{array}$ & $\begin{array}{c}96.8 \\
01 / 25 / 90\end{array}$ \\
\hline
\end{tabular}

notes: a) Sample moist, good condition. 


\begin{tabular}{|c|c|c|c|c|}
\hline $\begin{array}{l}\text { PSOC-1522 } \\
\text { collection date: }\end{array}$ & $\begin{array}{l}\text { EWELLL } \\
8 / 05 / 86\end{array}$ & mvb, & Ro $\max =1$ & 28 \\
\hline $\begin{array}{r}\text { Split ID } \\
\text { size } \\
\text { \% head oxygen } \\
\text { note } \\
\text { date sealed } \\
\text { date oxygen }\end{array}$ & $\begin{array}{l}\text { analysis } \\
\text { sample } 1 \\
-60 \text { mesh }\end{array}$ & $\begin{array}{l}\# 2 \text { can } \\
15 \text { of } 31 \\
-20 \text { mesh } \\
09 / 06 / 86\end{array}$ & $\begin{array}{c}30 \mathrm{gal} \\
3 \text { of } 3 \\
-1 / 4^{11} \\
19.1 \\
a \\
08 / 11 / 86 \\
10 / 28 / 88\end{array}$ & $\begin{array}{l}30 \text { gal. } \\
3 \text { of } 3 \\
-1 / 4^{\prime \prime} \\
20.3 \\
10 / 23 / 89\end{array}$ \\
\hline $\begin{array}{l}\text { PROXIMATE } \\
\% \text { moisture } \\
\% \text { ash (dry) } \\
\text { analysis date } \\
\end{array}$ & $\begin{array}{r}2.5 \\
18.8 \\
09 / 16 / 86 \\
\end{array}$ & $\begin{array}{r}1.3 \\
18.9 \\
05 / 25 / 88 \\
\end{array}$ & $\begin{array}{r}2.4 \\
17.9 \\
03 / 07 / 89 \\
\end{array}$ & $\begin{array}{r}3.5 \\
18.3 \\
03 / 01 / 90 \\
\end{array}$ \\
\hline $\begin{array}{l}\text { GIESELER FLUIDITY } \\
\text { max. ddpm } \\
\text { fluid range } \\
\text { analysis date }\end{array}$ & $\begin{array}{r}223 \\
72 \\
09 / 17 / 86 \\
\end{array}$ & $\begin{array}{r}6 \\
50 \\
05 / 25 / 88 \\
\end{array}$ & $\begin{array}{c}4 \\
45 \\
03 / 03 / 89\end{array}$ & $\begin{array}{l}0 \\
0\end{array}$ \\
\hline $\begin{array}{l}\text { SULFUR FORMS (dry } \% \text { ) } \\
\text { total sulfur } \\
\text { sulfate } \\
\text { pyritic } \\
\text { organic } \\
\text { analysis date } \\
\end{array}$ & $\begin{array}{c}1.17 \\
0.10 \\
0.42 \\
0.66 \\
09 / 17 / 86 \\
\end{array}$ & & $\begin{array}{c}1.05 \\
0.43 \\
0.16 \\
0.45 \\
03 / 07 / 89 \\
\end{array}$ & $\begin{array}{l}1.19 \\
0.40 \\
0.16 \\
0.63 \\
03 / 01 / 90\end{array}$ \\
\hline $\begin{array}{l}\text { CALORIFIC VALUE (dry) } \\
\text { BTU/lb } \\
\text { analys is date }\end{array}$ & $\begin{array}{c}12355 \\
09 / 17 / 86 \\
\end{array}$ & & $\begin{array}{c}12229 \\
03 / 07 / 89\end{array}$ & $\begin{array}{c}12258 \\
03 / 01 / 90\end{array}$ \\
\hline $\begin{array}{l}\text { ALKALI EXTRACTION } \\
\% \text { transmission } \\
\text { anaiysis date }\end{array}$ & & $\begin{array}{c}98.2 \\
06 / 10 / 88\end{array}$ & $\begin{array}{c}98.9 \\
03 / 06 / 89\end{array}$ & $\begin{array}{c}95.8 \\
02 / 15 / 90\end{array}$ \\
\hline
\end{tabular}

notes: a) Sample dry, good condition. 


\begin{tabular}{|c|c|c|c|c|}
\hline $\begin{array}{l}\text { PSOC-1523 } \\
\text { collection date: }\end{array}$ & $\begin{array}{l}5 \text { BLOCK } \\
8 / 06 / 86\end{array}$ & hvBb, & Ro $\max =0$ & \\
\hline $\begin{array}{r}\text { Split ID } \\
\text { size } \\
\text { \% head oxygen } \\
\text { note } \\
\text { date sealed } \\
\text { date oxygen }\end{array}$ & $\begin{array}{l}\text { analysis } \\
\text { sample } 1 \\
-60 \text { mesh }\end{array}$ & $\begin{array}{l}\# 2 \text { can } \\
15 \text { of } 31 \\
-20 \text { mesh } \\
09 / 08 / 86\end{array}$ & $\begin{array}{c}30 \mathrm{gal} \\
3 \text { of } 3 \\
-1 / 4^{\prime \prime} \\
20.1 \\
\text { a } \\
11 / 16 / 88\end{array}$ & $\begin{array}{l}30 \text { gal. } \\
3 \text { of } 3 \\
-1 / 4^{11} \\
20.4 \\
10 / 23 / 89\end{array}$ \\
\hline $\begin{array}{l}\text { PROXIMATE } \\
\% \text { molsture } \\
\% \text { ash (dry) } \\
\text { analysis date }\end{array}$ & $\begin{array}{c}2.6 \\
16.1 \\
09 / 25 / 86 \\
\end{array}$ & $\begin{array}{c}2.7 \\
14.5 \\
05 / 26 / 88 \\
\end{array}$ & $\begin{array}{c}2.5 \\
15.6 \\
03 / 07 / 89 \\
\end{array}$ & $\begin{array}{r}2.9 \\
15.9 \\
02 / 28 / 90 \\
\end{array}$ \\
\hline $\begin{array}{l}\text { GIESELER FLUIDITY } \\
\text { max. dopm } \\
\text { fluid range } \\
\text { analysis date }\end{array}$ & $\begin{array}{r}415 \\
58 \\
09 / 18 / 86\end{array}$ & $\begin{array}{c}10 \\
49 \\
05 / 26 / 88 \\
\end{array}$ & $\begin{array}{c}5 \\
33 \\
03 / 01 / 89 \\
\end{array}$ & $\begin{array}{c}3 \\
30 \\
02 / 16 / 90\end{array}$ \\
\hline $\begin{array}{l}\text { SULFUR FORMS (dry \%) } \\
\text { total sulfur } \\
\text { sulfate } \\
\text { pyritic } \\
\text { organic } \\
\text { analysis date }\end{array}$ & $\begin{array}{c}0.71 \\
0.00 \\
0.02 \\
0.69 \\
09 / 25 / 86\end{array}$ & & $\begin{array}{c}0.68 \\
0.06 \\
0.04 \\
0.57 \\
03 / 07 / 89 \\
\end{array}$ & $\begin{array}{c}0.72 \\
0.06 \\
0.03 \\
0.63 \\
02 / 28 / 90 \\
\end{array}$ \\
\hline $\begin{array}{l}\text { CALORIFIC VALUE (dry) } \\
\text { BTU/Ib } \\
\text { analysis date }\end{array}$ & $\begin{array}{c}12425 \\
09 / 25 / 86\end{array}$ & & $\begin{array}{c}12391 \\
03 / 07 / 89 \\
\end{array}$ & $\begin{array}{c}12340 \\
02 / 28 / 90 \\
\end{array}$ \\
\hline $\begin{array}{c}\text { ALKALI EXTRACTION } \\
\% \text { transmission } \\
\text { analysis date }\end{array}$ & & $\begin{array}{c}96.3 \\
06 / 10 / 88\end{array}$ & $\begin{array}{c}95.5 \\
03 / 06 / 89\end{array}$ & $\begin{array}{c}90.0 \\
02 / 16 / 90\end{array}$ \\
\hline
\end{tabular}

notes: a) Sample dry, good condition. 


\begin{tabular}{|c|c|c|c|c|}
\hline \multicolumn{5}{|c|}{$\begin{array}{l}\text { PSOC }-1524 \quad \text { UPPER KITTANNING hVAb, Ro } \max =1.06 \\
\text { collection date: } 10 / 23 / 86\end{array}$} \\
\hline $\begin{array}{r}\text { Split ID } \\
\text { size } \\
\% \text { head oxygen } \\
\text { note } \\
\text { date sealed } \\
\text { date oxygen }\end{array}$ & $\begin{array}{l}\text { analysis } \\
\text { sample } 1 \\
-60 \text { mesh }\end{array}$ & $\begin{array}{l}\# 2 \text { Can } \\
15 \text { of } 30 \\
-20 \text { mesh } \\
11 / 04 / 86\end{array}$ & $\begin{array}{c}30 \text { gal } \\
3 \text { of } 3 \\
-1 / 4^{\prime \prime} \\
20.3 \\
\text { a } \\
11 / 16 / 88\end{array}$ & $\begin{array}{l}30 \text { ga } 1 \\
3 \text { of } 3 \\
-1 / 4^{11} \\
20.4 \\
10 / 24 / 89\end{array}$ \\
\hline $\begin{array}{l}\text { PROXIMATE } \\
\% \text { moisture } \\
\% \text { ash (dry) } \\
\text { analysis date }\end{array}$ & $\begin{array}{c}1.6 \\
11.0 \\
11 / 25 / 86\end{array}$ & $\begin{array}{c}1.5 \\
11.5 \\
05 / 31 / 88\end{array}$ & $\begin{array}{c}1.8 \\
10.9 \\
03 / 07 / 89\end{array}$ & $\begin{array}{l}2.0 \\
11.5 \\
02 / 28 / 90\end{array}$ \\
\hline $\begin{array}{l}\text { GIESELER FLUIDITY } \\
\text { max. ddpm } \\
\text { fluid range } \\
\text { analys is date } \\
\end{array}$ & $\begin{array}{c}30000 \\
110 \\
03 / 17 / 87 \\
\end{array}$ & $\begin{array}{r}4589 \\
91 \\
05 / 31 / 88\end{array}$ & $\begin{array}{c}3907 \\
88 \\
03 / 03 / 89\end{array}$ & $\begin{array}{c}752 \\
82 \\
02 / 19 / 90\end{array}$ \\
\hline $\begin{array}{l}\text { SULFUR FORMS (dry \%) } \\
\text { total sulfur } \\
\text { sulfate } \\
\text { pyritic } \\
\text { organic } \\
\text { analysis date } \\
\end{array}$ & $\begin{array}{r}1.85 \\
0.02 \\
1.39 \\
0.44 \\
11 / 25 / 86 \\
\end{array}$ & & $\begin{array}{c}1.76 \\
0.18 \\
1.08 \\
0.51 \\
03 / 07 / 89 \\
\end{array}$ & $\begin{array}{r}2.01 \\
0.25 \\
0.93 \\
0.83 \\
02 / 28 / 90\end{array}$ \\
\hline $\begin{array}{l}\text { CALORIFIC VALUE (dry) } \\
\text { BTU/lb } \\
\text { analysis date }\end{array}$ & $\begin{array}{c}13656 \\
11 / 17 / 86 \\
\end{array}$ & & $\begin{array}{r}13469 \\
03 / 07 / 89 \\
\end{array}$ & $\begin{array}{c}13378 \\
02 / 23 / 90\end{array}$ \\
\hline $\begin{array}{c}\text { ALKALI EXTRACTION } \\
\% \text { transmission } \\
\text { analysis date }\end{array}$ & & $\begin{array}{c}98.3 \\
06 / 10 / 88 \\
\end{array}$ & $\begin{array}{c}98.2 \\
03 / 08 / 89\end{array}$ & $\begin{array}{r}93.5 \\
02 / 19 / 90 \\
\end{array}$ \\
\hline
\end{tabular}

notes: a) Sample dry, good condition. 


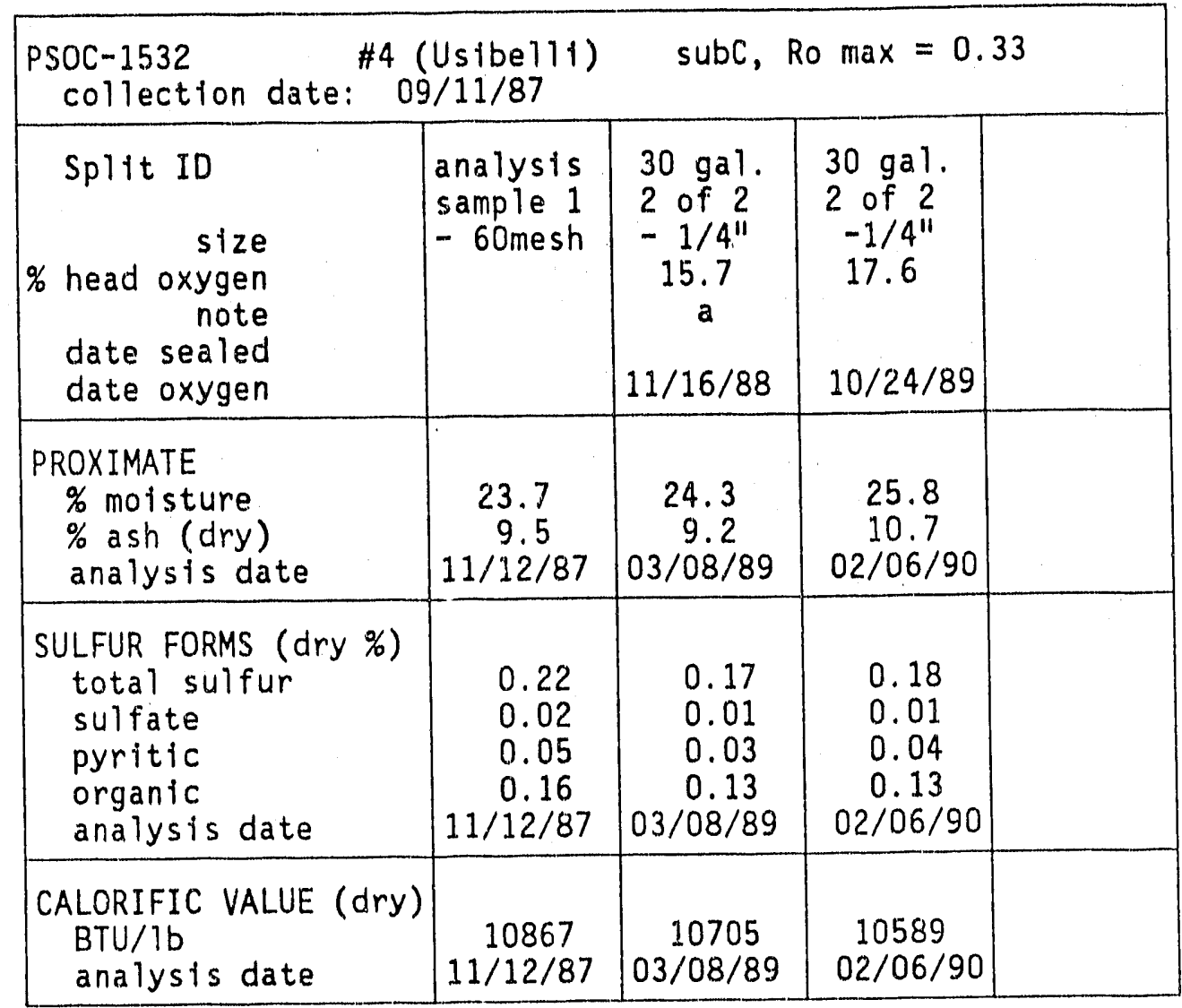

notes: a) Sample molst, good condition 


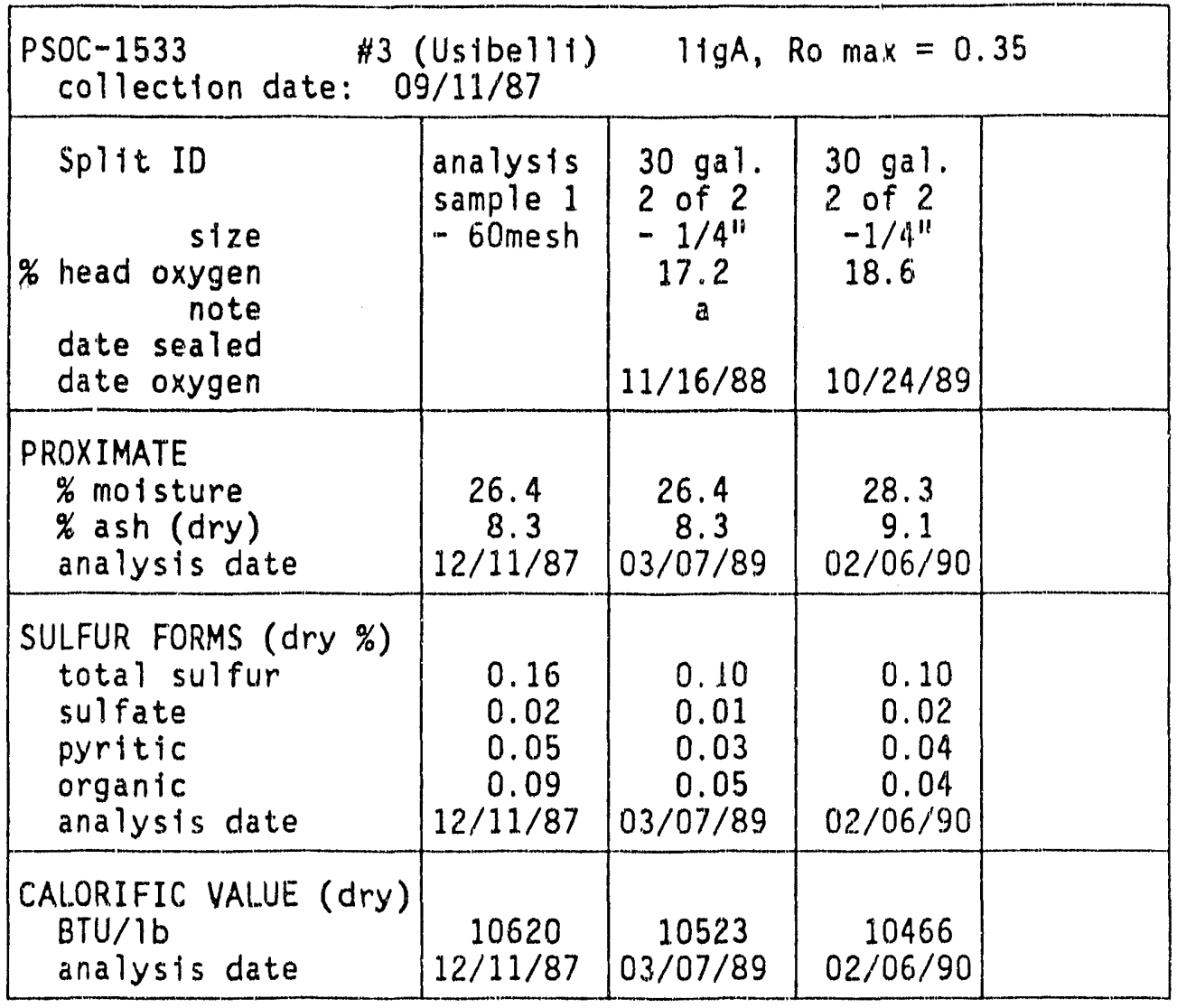

notes: a) Sample moist, good condition. 


\begin{tabular}{|c|c|c|c|c|}
\hline $\begin{array}{l}\text { PSOC- } 1534 \\
\text { collection date: }\end{array}$ & $\begin{array}{l}\text { NATERFALL } \\
99 / 14 / 87\end{array}$ & subc, & $0 \max =0$ & \\
\hline $\begin{array}{r}\text { Split ID } \\
\text { size } \\
\% \text { head oxygen } \\
\text { note } \\
\text { date sealed } \\
\text { date oxygen }\end{array}$ & $\begin{array}{l}\text { analysis } \\
\text { sample } 1 \\
-60 \text { mesh }\end{array}$ & $\begin{array}{l}30 \mathrm{gal} \\
2 \text { of } 2 \\
-1 / 4^{\prime \prime} \\
13.6 \\
\mathrm{a} \\
11 / 16 / 88\end{array}$ & $\begin{array}{l}30 \text { gal. } \\
2 \text { of } 2 \\
-1 / 4^{\prime \prime} \\
15.2 \\
10 / 24 / 89\end{array}$ & \\
\hline $\begin{array}{l}\text { PROXIMATE } \\
\% \text { moisture } \\
\% \text { ash (dry) } \\
\text { analysis date } \\
\end{array}$ & $\begin{array}{c}21.8 \\
11.1 \\
11 / 12 / 87 \\
\end{array}$ & $\begin{array}{l}23.1 \\
10.8\end{array}$ & $\begin{array}{c}23.8 \\
11.4 \\
02 / 06 / 90\end{array}$ & \\
\hline $\begin{array}{l}\text { SULFUR FORMS (dry \%) } \\
\text { total sulfur } \\
\text { sulfate } \\
\text { pyritic } \\
\text { organic } \\
\text { analysis date } \\
\end{array}$ & $\begin{array}{c}0.15 \\
0.01 \\
0.05 \\
0.09 \\
11 / 12 / 87\end{array}$ & $\begin{array}{c}0.13 \\
0.02 \\
0.01 \\
0.10 \\
03 / 07 / 89\end{array}$ & $\begin{array}{c}0.12 \\
0.01 \\
0.01 \\
0.09 \\
02 / 06 / 90\end{array}$ & \\
\hline $\begin{array}{l}\text { CALORIFIC VALLE (dry) } \\
\text { BTU/Ib } \\
\text { analysis date }\end{array}$ & $\begin{array}{c}11082 \\
11 / 12 / 87\end{array}$ & & $\begin{array}{c}10832 \\
02 / 06 / 90\end{array}$ & \\
\hline
\end{tabular}

notes: a) Sample moist, good condition. 


\begin{tabular}{|c|c|c|c|c|}
\hline \multicolumn{5}{|c|}{$\begin{array}{l}\text { PSOC- } 1535 \text { DEADFALL SYNCLINE \#4 subA, Ro max }=0.73 \\
\text { collection date: } 10 / 08 / 87\end{array}$} \\
\hline $\begin{array}{r}\text { Split ID } \\
\text { size } \\
\% \text { head oxygen } \\
\text { note } \\
\text { date sealed } \\
\text { date oxygen }\end{array}$ & $\begin{array}{l}\text { analysis } \\
\text { sample } 1 \\
-60 \text { mesh }\end{array}$ & $\begin{array}{l}\# 2 \text { Can } \\
-20 \text { mesh } \\
12 / 14 / 87\end{array}$ & $\begin{array}{c}30 \mathrm{gal} \\
2 \text { of } 2 \\
-1 / 4^{\prime \prime} \\
20.8 \\
\mathrm{a} \\
11 / 16 / 88\end{array}$ & $\begin{array}{l}30 \text { gal. } \\
2 \text { of } 2 \\
-1 / 4^{11} \\
19.2 \\
10 / 25 / 89\end{array}$ \\
\hline $\begin{array}{l}\text { PROXIMATE } \\
\% \text { moisture } \\
\% \text { ash (dry) } \\
\text { analysis date } \\
\end{array}$ & $\begin{array}{r}10.1 \\
3.3 \\
12 / 23 / 87 \\
\end{array}$ & $\begin{array}{r}10.2 \\
3.4 \\
06 / 14 / 88 \\
\end{array}$ & $\begin{array}{c}10.1 \\
3.4 \\
03 / 07 / 89\end{array}$ & $\begin{array}{r}10.6 \\
3.7 \\
02 / 06 / 90\end{array}$ \\
\hline $\begin{array}{l}\text { GIESELER FLUIDITY } \\
\text { max. ddpm } \\
\text { fluid range } \\
\text { analysis date } \\
\end{array}$ & $\begin{array}{l}0 \\
0\end{array}$ & $\begin{array}{l}\text { discon- } \\
\text { tinued }\end{array}$ & & \\
\hline $\begin{array}{l}\text { SUILFUR FORMS (dry } \% \text { ) } \\
\text { total sulfur } \\
\text { sulfate } \\
\text { pyritic } \\
\text { organic } \\
\text { analysis date }\end{array}$ & $\begin{array}{c}1.46 \\
0.02 \\
0.10 \\
1.35 \\
12 / 23 / 87 \\
\end{array}$ & & $\begin{array}{c}0.16 \\
0.01 \\
0.01 \\
0.14 \\
03 / 07 / 89 \\
\end{array}$ & $\begin{array}{r}0.13 \\
0.01 \\
0.01 \\
0.12 \\
02 / 06 / 90 \\
\end{array}$ \\
\hline $\begin{array}{l}\text { CALORIFIC VALUE (dry) } \\
\text { BTU/Ib } \\
\text { analysis date }\end{array}$ & $\begin{array}{c}12107 \\
12 / 23 / 87 \\
\end{array}$ & & $\begin{array}{c}12085 \\
03 / 07 / 89 \\
\end{array}$ & $\begin{array}{c}12074 \\
02 / 06 / 90 \\
\end{array}$ \\
\hline $\begin{array}{l}\text { ALKALI EXTRACTION } \\
\% \text { transmission } \\
\text { analysis date }\end{array}$ & & $\begin{array}{c}0.1 \\
06 / 16 / 88\end{array}$ & $\begin{array}{l}\text { discon- } \\
\text { tinued }\end{array}$ & \\
\hline
\end{tabular}

notes: a) Sample dry, good condition. 


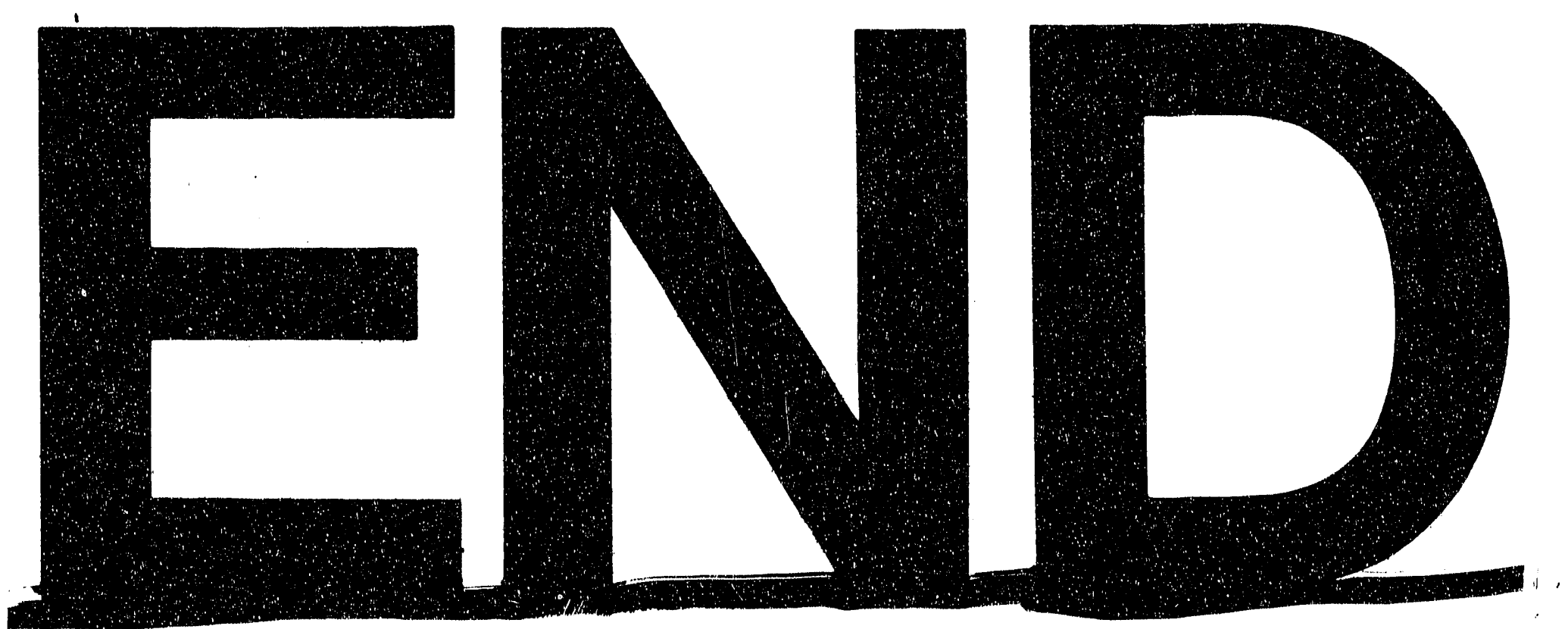

1
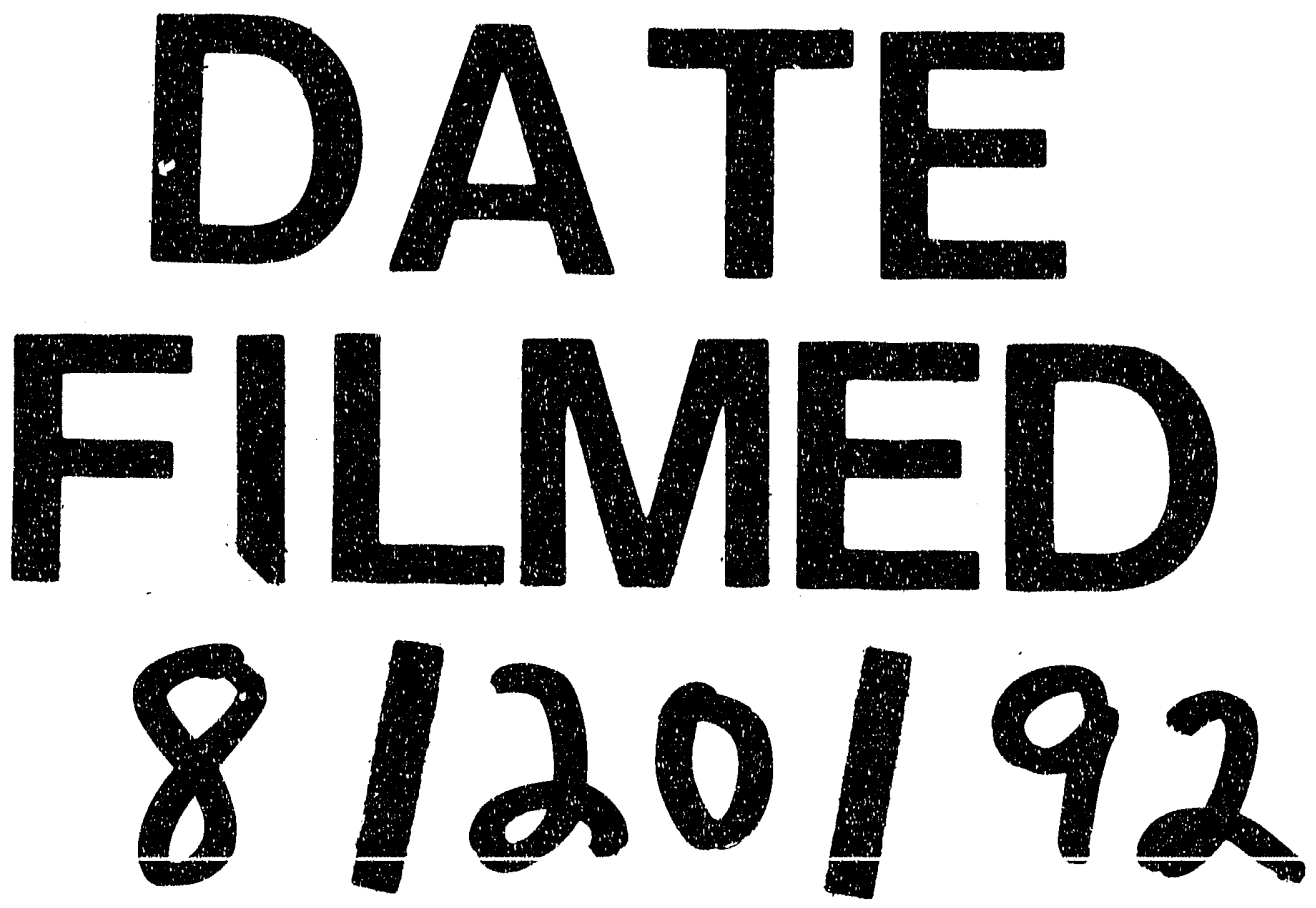
